\title{
Magnetic field installations and QED
}

\author{
Geert Rikken
}

Laboratoire National des Champs Magnétiques Intenses

Toulouse - France 


\section{Magnetic field installations and QED}

G. Rikken LNCMI-CNRS

Why magnetic fields

Magnetic field generation

state of the art

future developements

High magnetic field facilities

EMEC

current

future

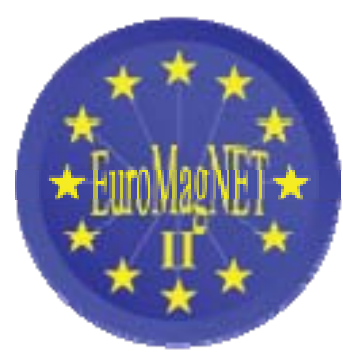

European Magnetic Field Laboratory 


\section{Why high magnetic fields I ?}

1) Manipulating matter: deflection levitation separation alignment

2) Probing matter: the field modifies electronic et magnetic properties:

Nuclear magnetic resonance (NMR, MRI)

'materials science' cyclotron resonance electron spin resonance (ESR)

Hall effect, dHvA effect, SdH effect,....

3) Thermo dynamics; Induce new states of matter:

LT superconductor normal state

'basic solid state physics' Field induced superconductivity Quantum critical points Magnetization plateau states 
Why high magnetic fields II ?

4) Symmetry

$$
\begin{aligned}
& \mathbf{B}_{0}=\frac{1}{4 \pi \varepsilon_{0}} \frac{2 \mathbf{I} \times \mathbf{r}}{r^{2}} \\
& \mathbf{I}=q N \frac{\partial \mathbf{r}}{\partial t}
\end{aligned}
$$

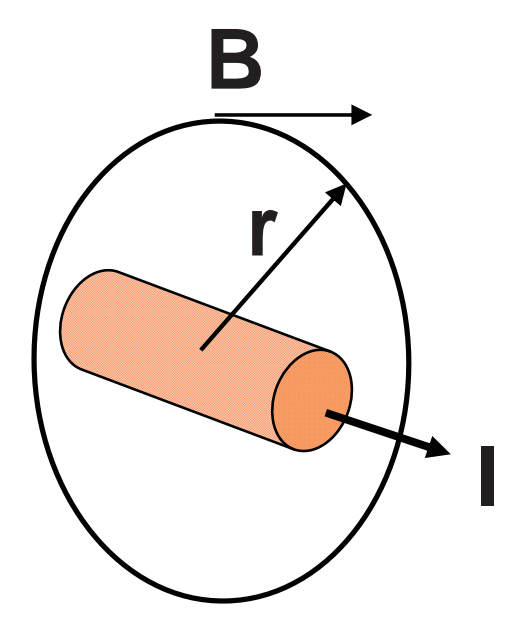

Parity reversal $\quad \mathrm{P}: \mathrm{r} \rightarrow-\mathrm{r}, \mathrm{t} \rightarrow \mathrm{t}, \mathrm{q} \rightarrow \mathrm{q}$ so $\mathrm{B} \rightarrow \mathrm{B}$ Charge conjugation $\mathrm{C}: \mathrm{r} \rightarrow \mathrm{r}, \mathrm{t} \rightarrow \mathrm{t}, \mathrm{q} \rightarrow-\mathrm{q}$ so $\mathrm{B} \rightarrow-\mathrm{B}$ Time reversal $\quad \mathrm{T}: \mathrm{r} \rightarrow \mathrm{r}, \mathrm{t} \rightarrow-\mathrm{t}, \mathrm{q} \rightarrow \mathrm{q}$ so $\mathrm{B} \rightarrow-\mathrm{B}$

Magnetic field is a time-odd pseudo-vector 
Symmetry properties of common physical quantities

\begin{tabular}{|l|c|c|c|c|}
\hline & & C & P & T \\
\hline polarization & P & - & - & + \\
\hline magnetization & M & - & + & - \\
\hline wavevector & $\mathbf{k}$ & + & - & - \\
\hline electrical current & I & - & - & - \\
\hline magnetic field & B & - & + & - \\
\hline electric field & E & - & - & + \\
\hline linear momentum & P & + & - & - \\
\hline angular momentum & L & + & + & - \\
\hline
\end{tabular}




\section{Symmetry and magneto-optics}

Perturbation approach:

$$
\mathbf{P}^{\omega}\left(\mathbf{B}_{0}\right)=\chi\left(\omega, \mathbf{B}_{0}\right) \mathbf{E}^{\omega} \simeq \chi(\omega) \mathbf{E}^{\omega}+f_{1}\left(\mathbf{B}_{0}, \mathbf{E}^{\omega}\right)+f_{2}\left(\mathbf{B}_{0}, \mathbf{B}_{0}, \mathbf{E}^{\omega}\right)+\ldots
$$

Symmetry:

\begin{tabular}{|c|c|c|c|}
\hline & C & P & T \\
\hline P & - & - & + \\
\hline B & - & + & - \\
\hline E & - & - & + \\
\hline
\end{tabular}

Only symmetry allowed form from $\mathrm{P}$ and $\mathrm{T}$ invariance:

$$
\begin{aligned}
\mathbf{P}^{\omega}\left(\mathbf{B}_{0}\right) & \simeq \chi(\omega) \mathbf{E}^{\omega}+\chi_{1} \frac{\partial \mathbf{E}^{\omega}}{\partial t} \times \mathbf{B}_{0}+\chi_{2}\left(\mathbf{B}_{0} \cdot \mathbf{B}_{0}\right) \mathbf{E}^{\omega}+\chi_{3}\left(\mathbf{B}_{0} \cdot \mathbf{E}^{\omega}\right) \mathbf{B}_{0}+\ldots . \\
& \simeq \chi(\omega) \mathbf{E}^{\omega}+i \omega \chi_{1} \mathbf{B}_{0} \times \mathbf{E}^{\omega}+O\left(B_{0}^{2}\right)
\end{aligned}
$$

Dielectric constant: $\quad \varepsilon_{i j}\left(\omega, \mathbf{B}_{0}\right)=1+4 \pi \chi_{i j} \simeq \varepsilon_{i j}(\omega)+i \gamma(\omega) e_{i j k} B_{0 k}$

$\gamma(\omega)$ odd under $\mathbf{C}$ 
Maxwell:

$$
\nabla \times \nabla \times \mathbf{E}^{\omega}=-\frac{\varepsilon}{c} \frac{\partial^{2} \mathbf{E}^{\omega}}{\partial t^{2}}
$$

Faraday geometry: $\quad \mathbf{k} \uparrow \uparrow \mathbf{B}_{0} \equiv \mathbf{z}$

Ansatz: $\quad \mathbf{E}^{\omega}=E^{\omega} \exp (i \omega t-i k z) \mathbf{x}$

Solution: $\mathbf{E}^{\omega}(z)=E^{\omega} \exp (i \omega t)\left\{\exp \left(-i k_{+} z\right) \mathbf{e}_{+}+\exp \left(-i k_{-} z\right) \mathbf{e}_{-}\right\}$

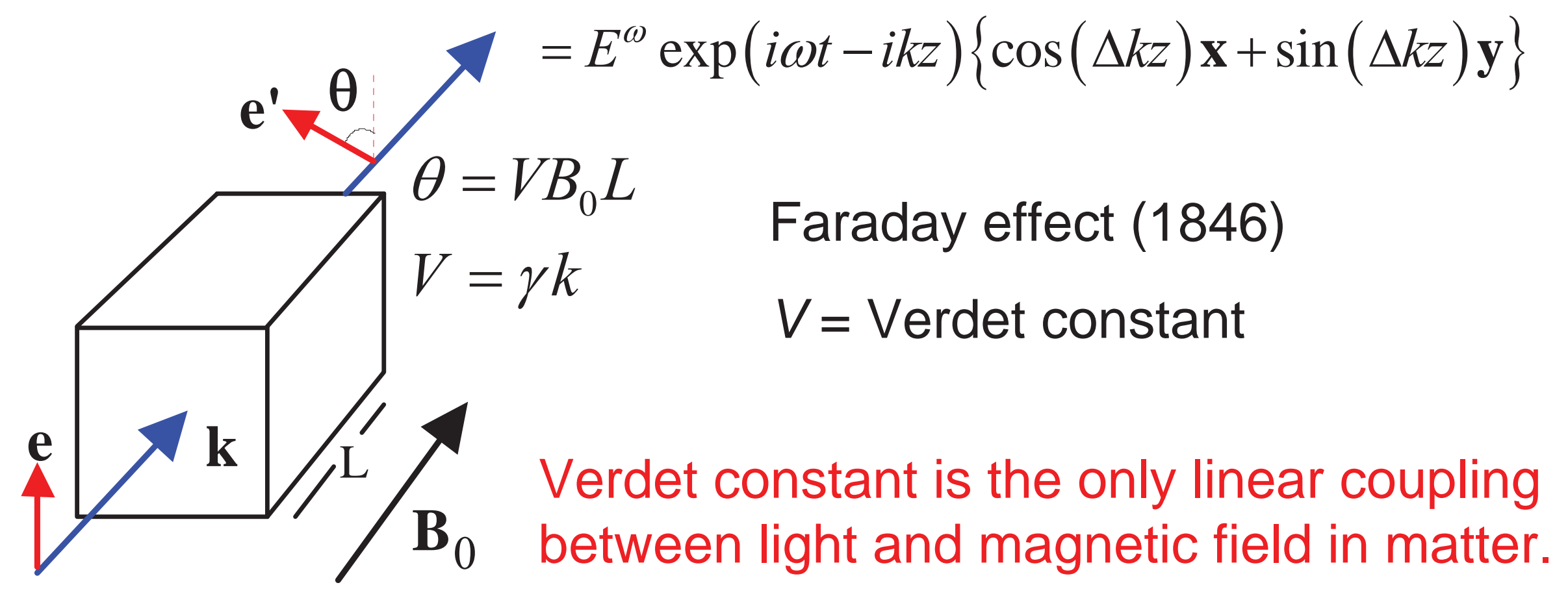




\section{Why high magnetic fields III?}

5) Quantum mechanics

Hamiltonian: $\mathbf{p} \rightarrow \mathbf{p}+\mathbf{q} \mathbf{A}$ and $\mathbf{B}=\nabla \times \mathbf{A}$

Energy/time scale: $\quad \hbar \omega_{c}=\hbar e B / m=100 \mu \mathrm{eV} \cdot B$

(Cyclotron energy)

$$
\hbar \omega_{Z}=g \mu_{B} B=100 \mu e V \cdot B
$$

Length scale:

$$
\ell=\sqrt{e \hbar / B}=25,6 \mathrm{~nm} / \sqrt{B}
$$




\section{Why high magnetic fields III?}

6) Quantum electrodynamics

Critical magnetic field

$$
\hbar \omega_{c}=\hbar e B_{c r} / m=m c^{2} \rightarrow B_{c r}=4,4 \cdot 10^{9} \mathrm{~T}
$$

Magnetic field induced QED effects in the laboratory will be weak and therefore a considerable experimental challenge!

E.g. vacuum polarization: $n(B)-1=\frac{\alpha}{6 \pi}\left(B / B_{c r}\right)^{2} \approx 10^{-23} B^{2}$ 
Why high magnetic fields IV?

7) Beyond the standard model ???

Axion-photon conversion 


\section{Nobel prizes for magnetic field research}

1902 Physics H.A. Lorentz \& P. Zeeman: Magnetic effects on radiation

1922 Chemistry F. Aston: Mass spectrometer

1939 Physics E. Lawrence: Development of the cyclotron

1943 Physics O. Stern: Magnetic moment of the proton

1944 Physics I. Rabi: NMR of atoms and molecules

1952 Physics F. Bloch, E. Purcell: Condensed matter NMR

1955 Physics P. Kusch: Measurement of the electron magnetic moment

1970 Physics L. Neel: Anti-ferromagnetism, ferrimagnetism

1977 Physics Anderson, Mott, van Vleck: Magnetic and disordered systems

1985 Physics K. von Klitzing: Quantum Hall effect

1991 Chemistry R. Ernst: 2D and FT NMR

1998 Physics Laughlin, Stormer, Tsui: Fractional quantum Hall effect

2002 Chemistry K. Wuthrich: NMR of biological macromolecules

2003 Medecine P. Lauterbur, P. Mansfield: Magnetic resonance imaging

2007 Physics Fert \& Grunberg, Giant magneto-resistance 


\section{Orders of magnitude of magnetic fields}

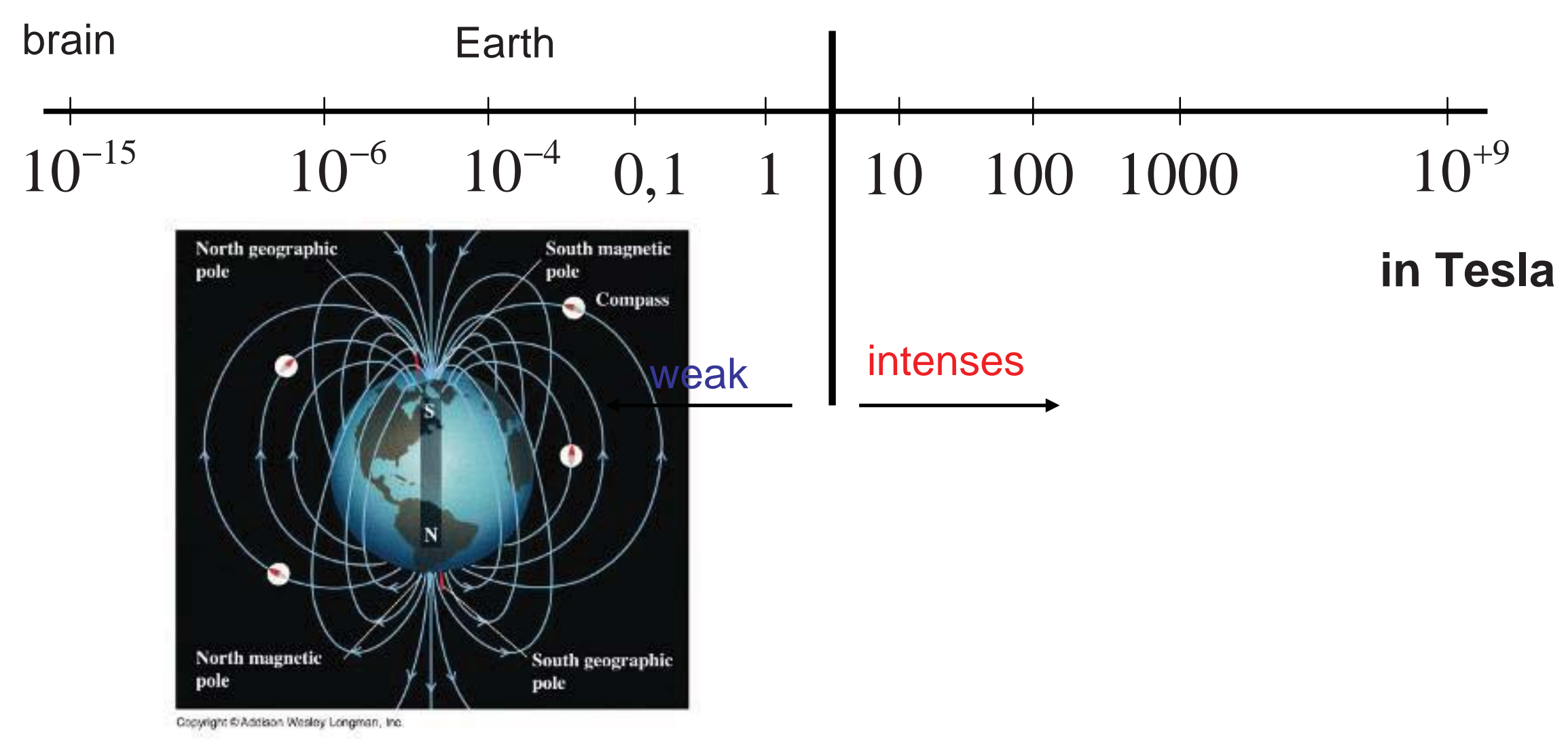




\section{Orders of magnitude of magnetic fields}

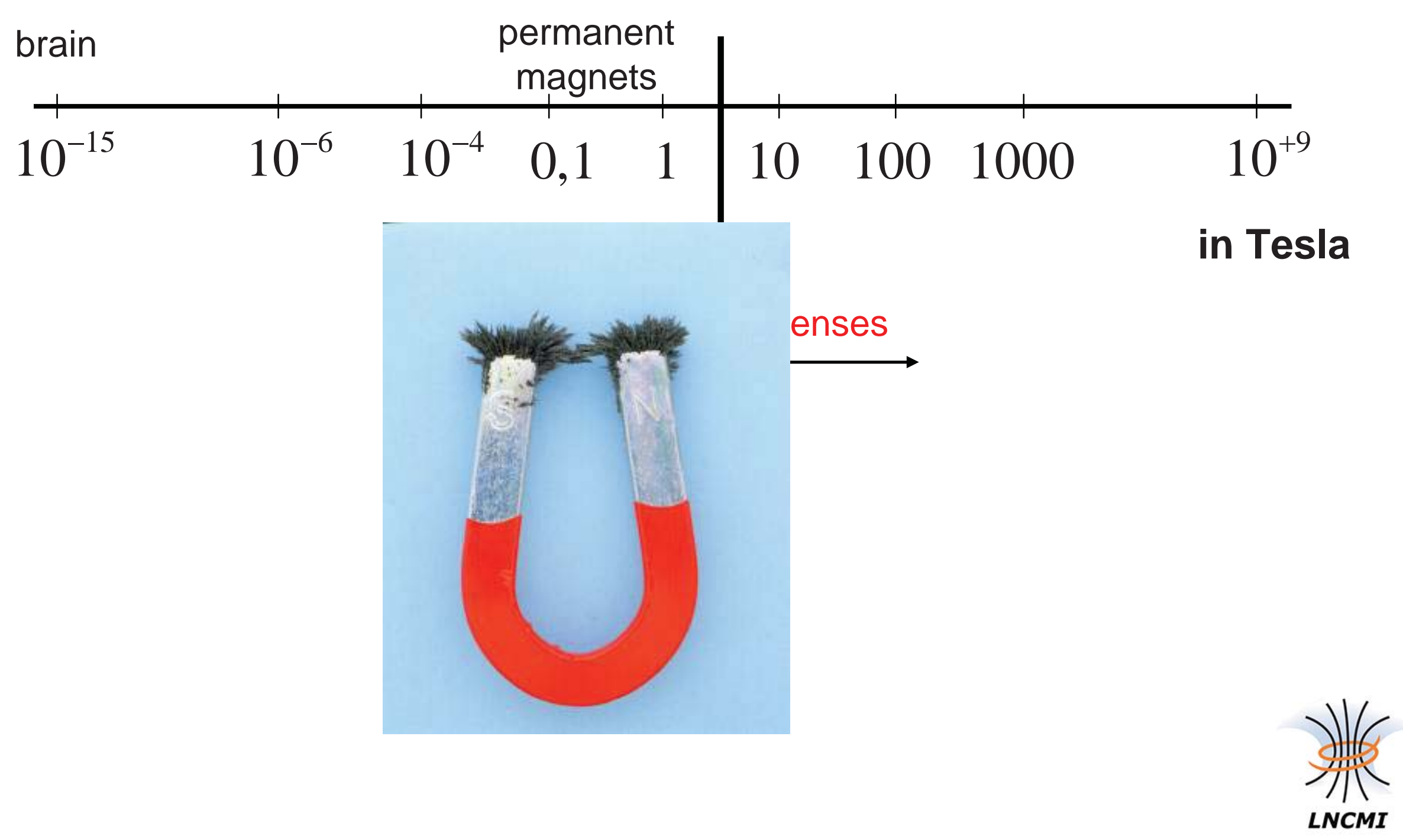




\section{Orders of magnitude of magnetic fields}

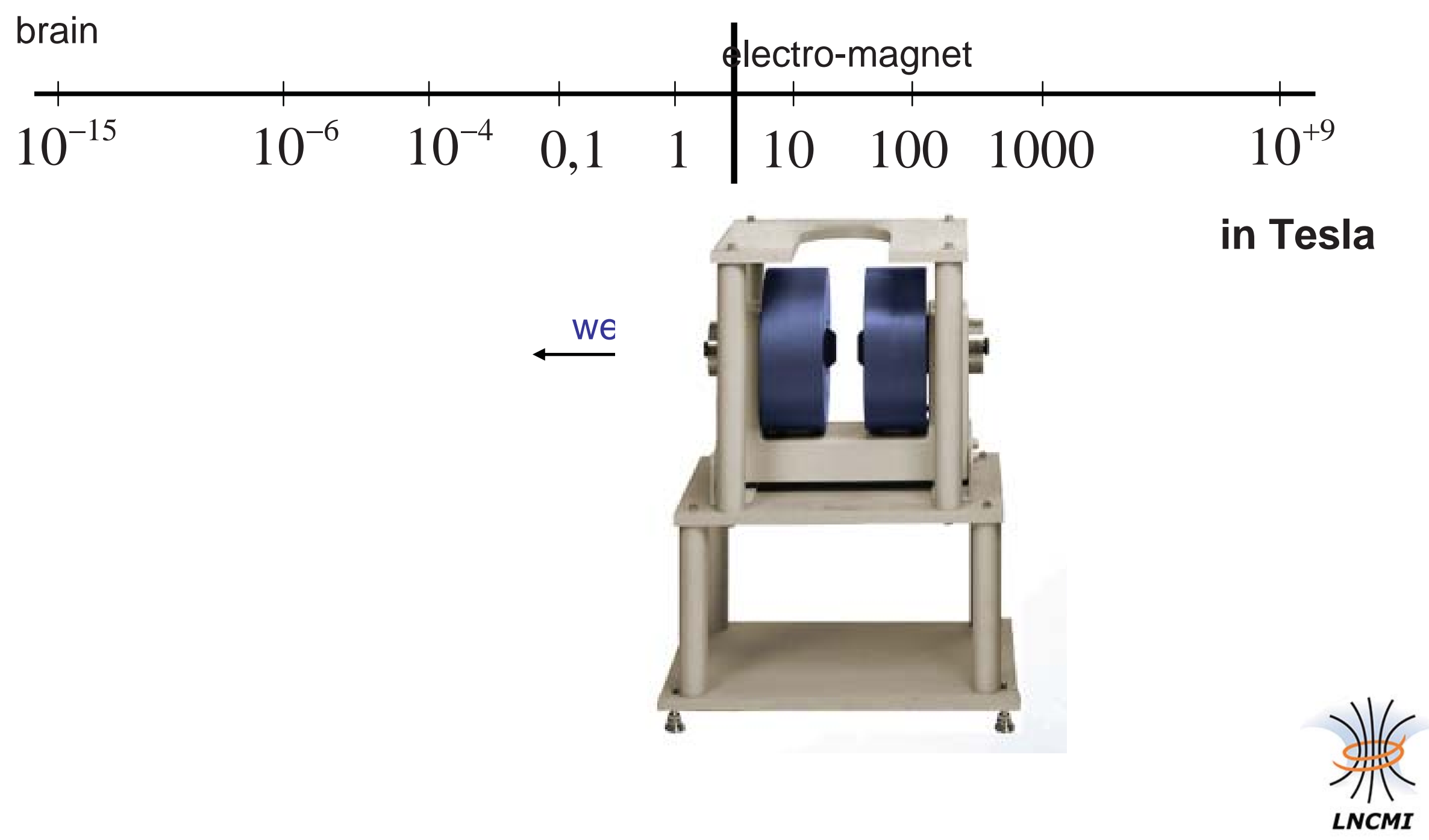




\section{Orders of magnitude of magnetic fields}

magnetar

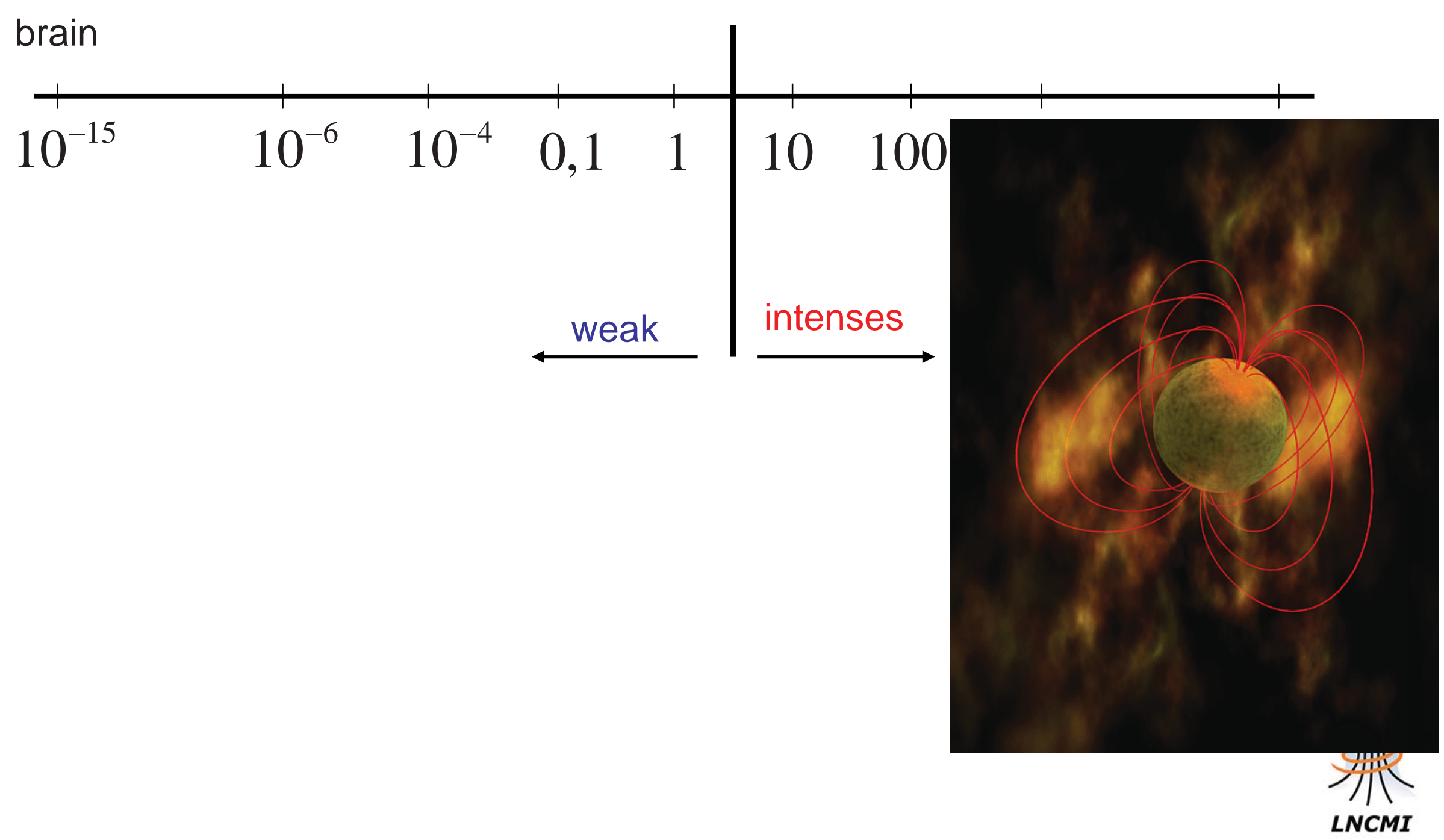


How to generate high magnetic fields for general use?

One solution: circulate a current I in a coil:

$$
\mathrm{B} \propto \mathrm{I}
$$

Problem 1: $\quad$ heating $\mathrm{P} \propto \mathrm{RI}^{2} \propto \mathrm{B}^{2}$

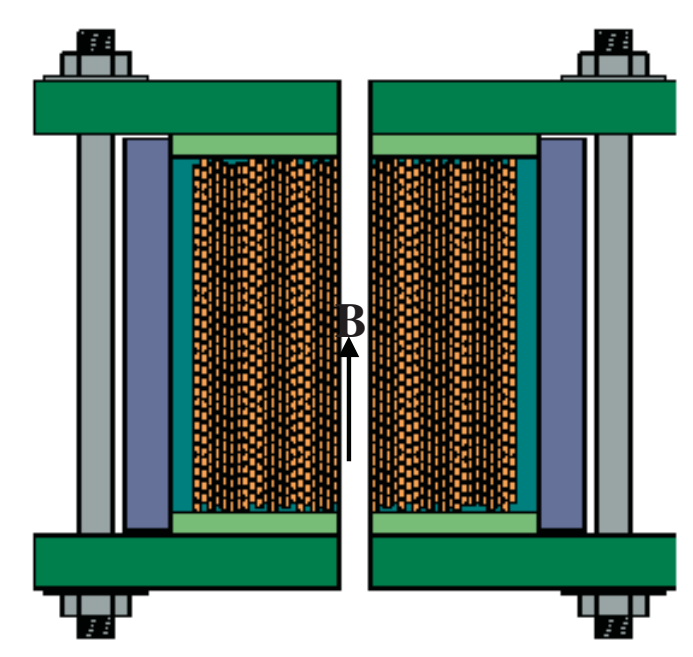

Solutions: $\quad$ - superconductors $(\mathrm{R}=0)$

- cooling: DC fields

- short current pulse $(<1 \mathrm{~s})$ : pulsed field

Problem 2: $\quad$ Lorentz forces $\propto \mathbf{B} \mathbf{X I} \propto \mathrm{B}^{2}$

Solutions: - strong conductor

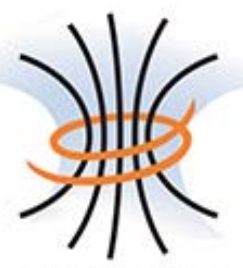

- mecanical reinforcement

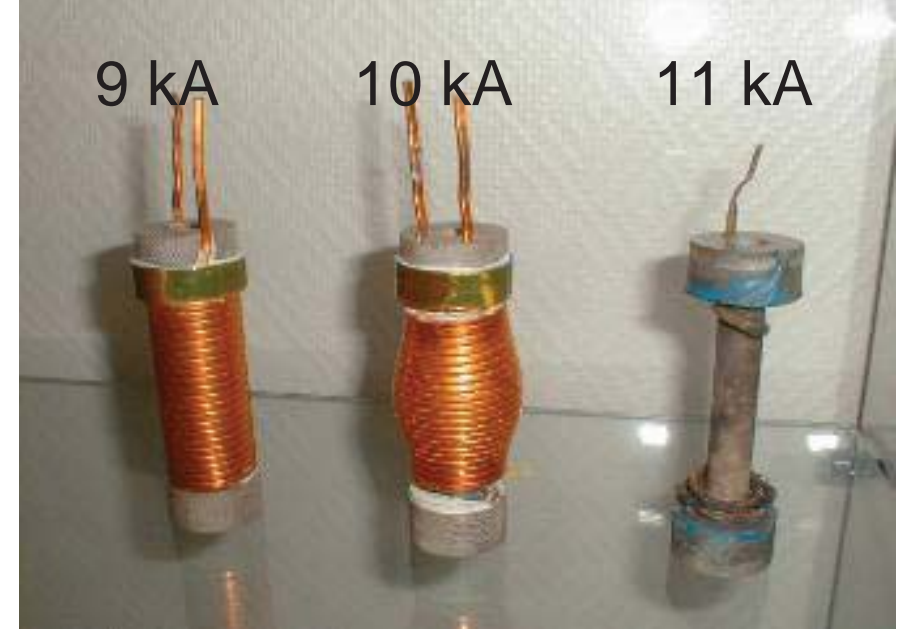




\section{Magnetic fields for research}

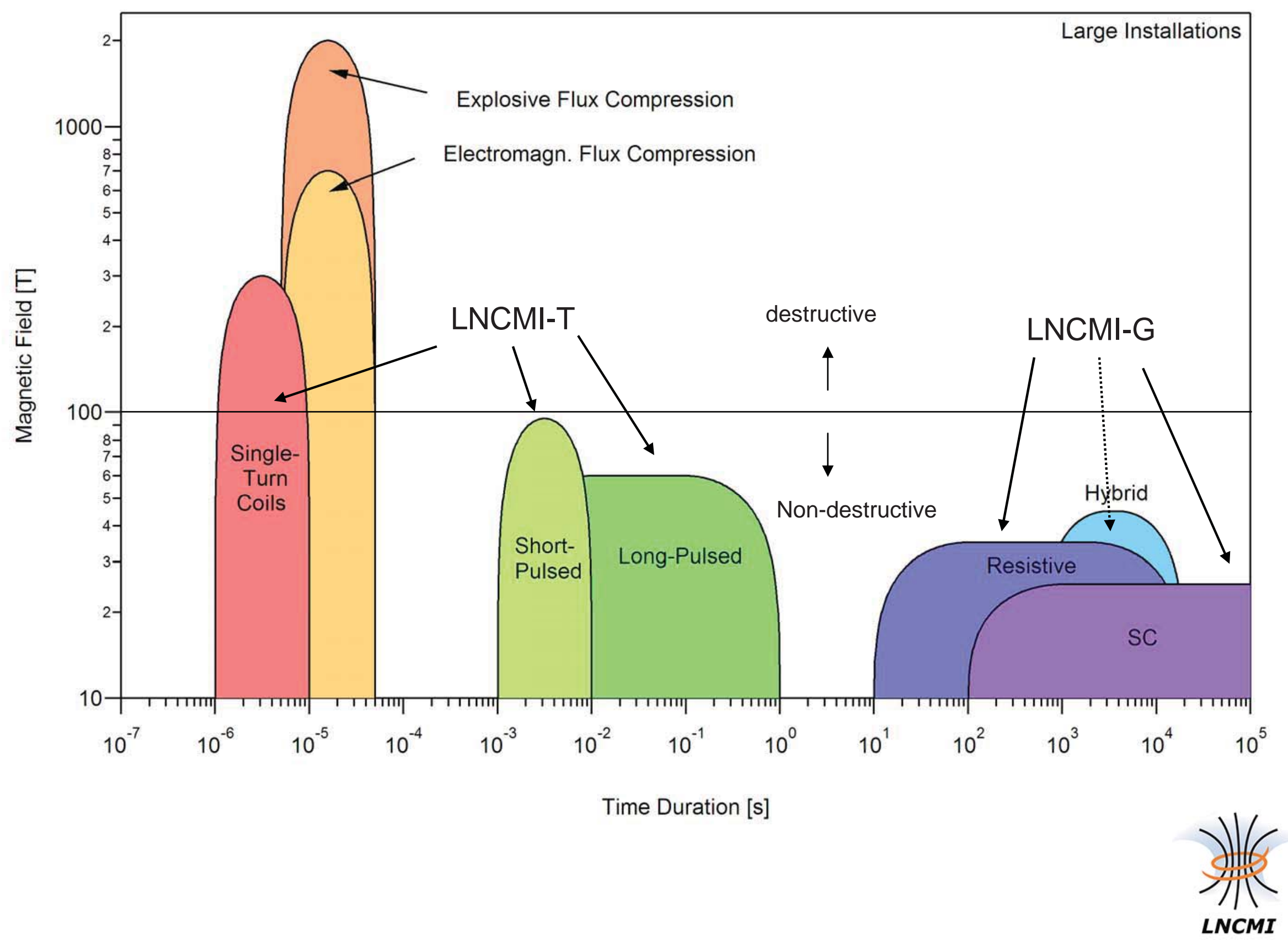


Evolution non-destructive high magnetic field generation methods

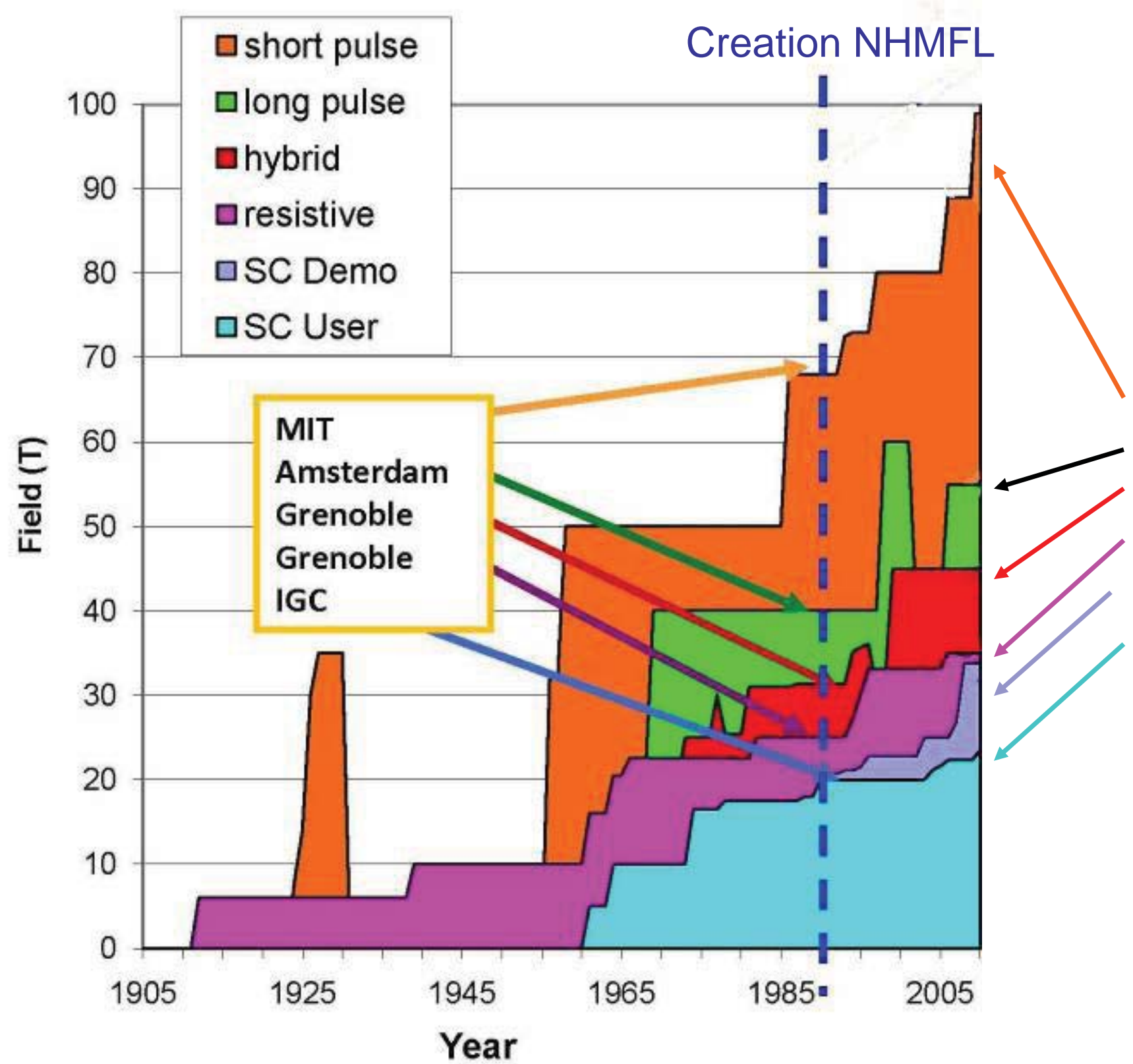

NHMFL

NHMFL

NHMFL

NHMFL

NHMFL

Bruker

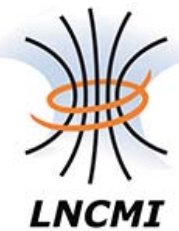


Static magnetic fields

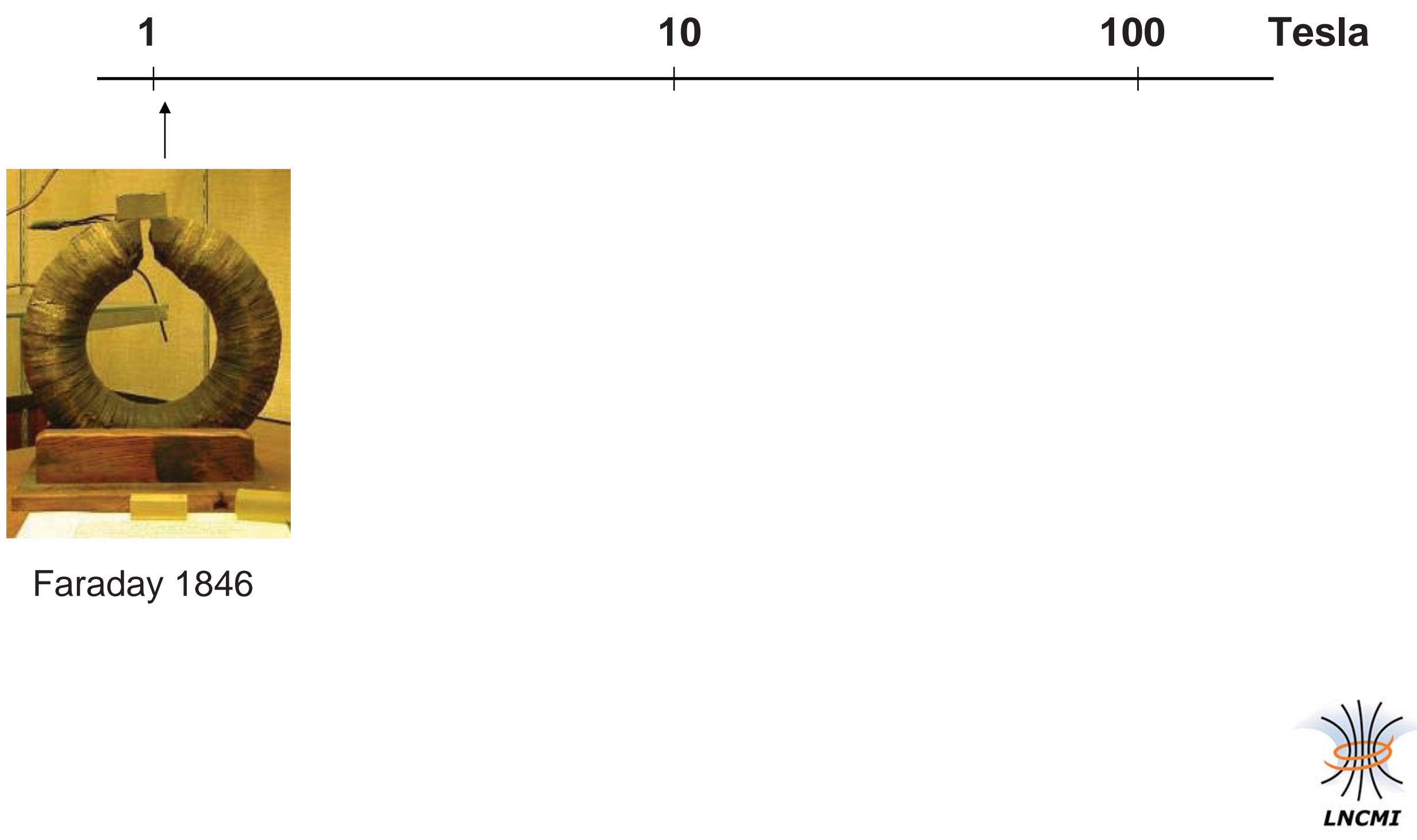


Static magnetic fields

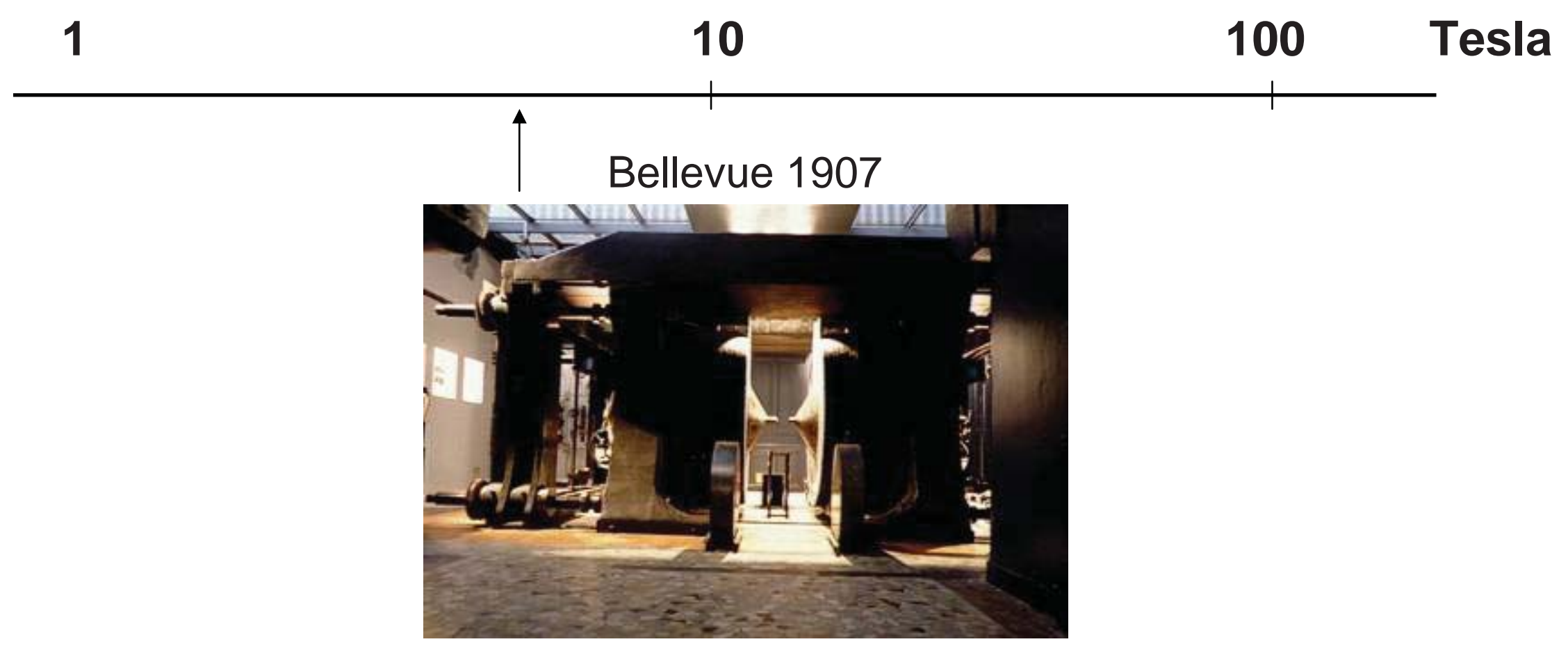


Static magnetic fields

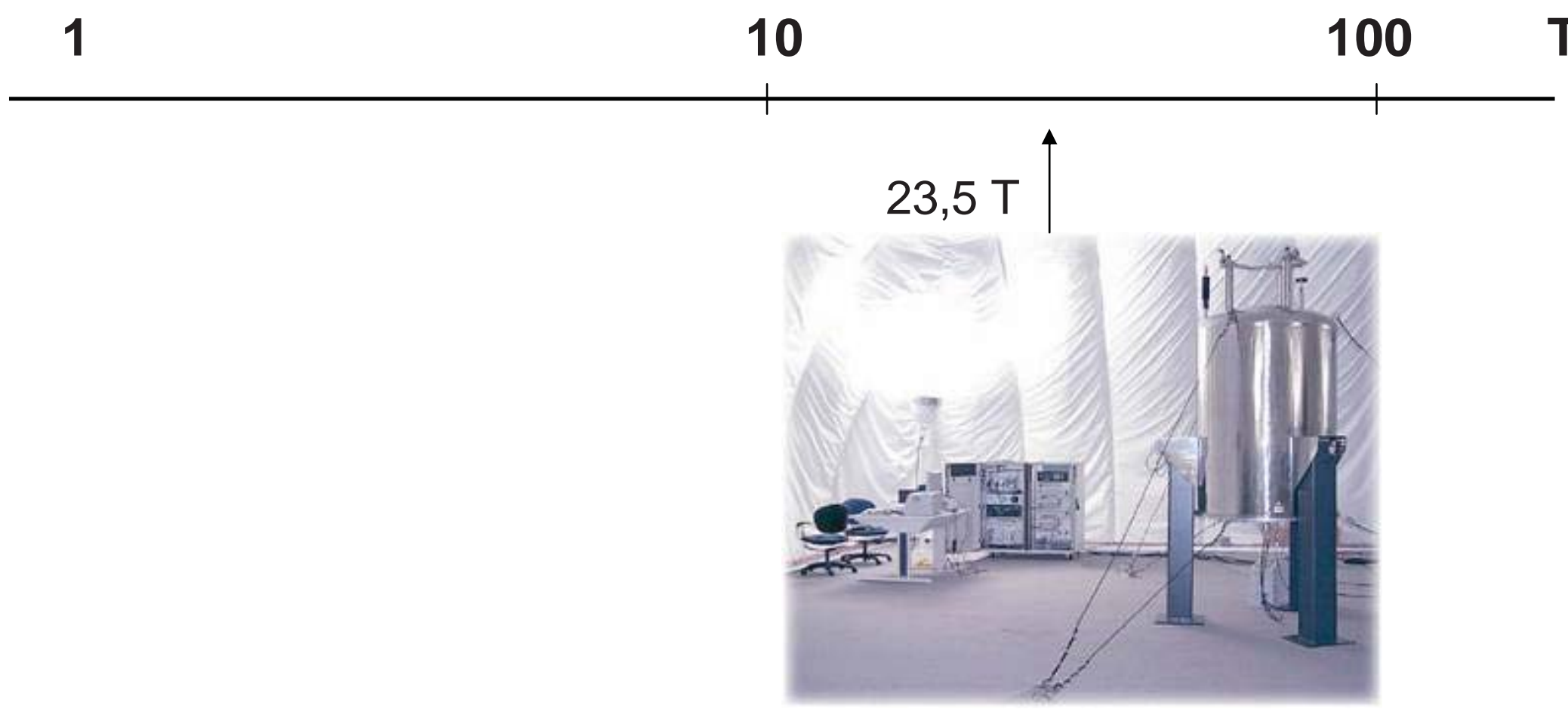


Static magnetic fields

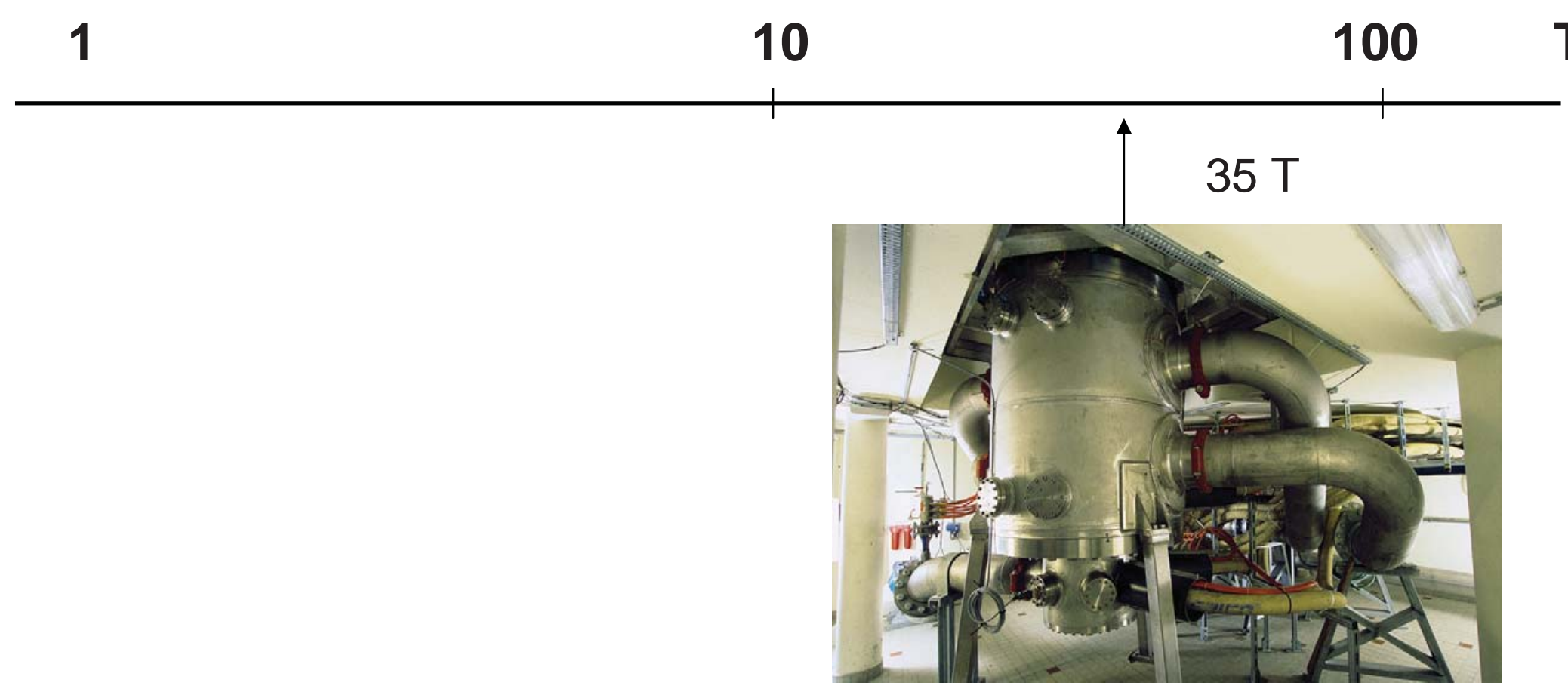


Static magnetic fields

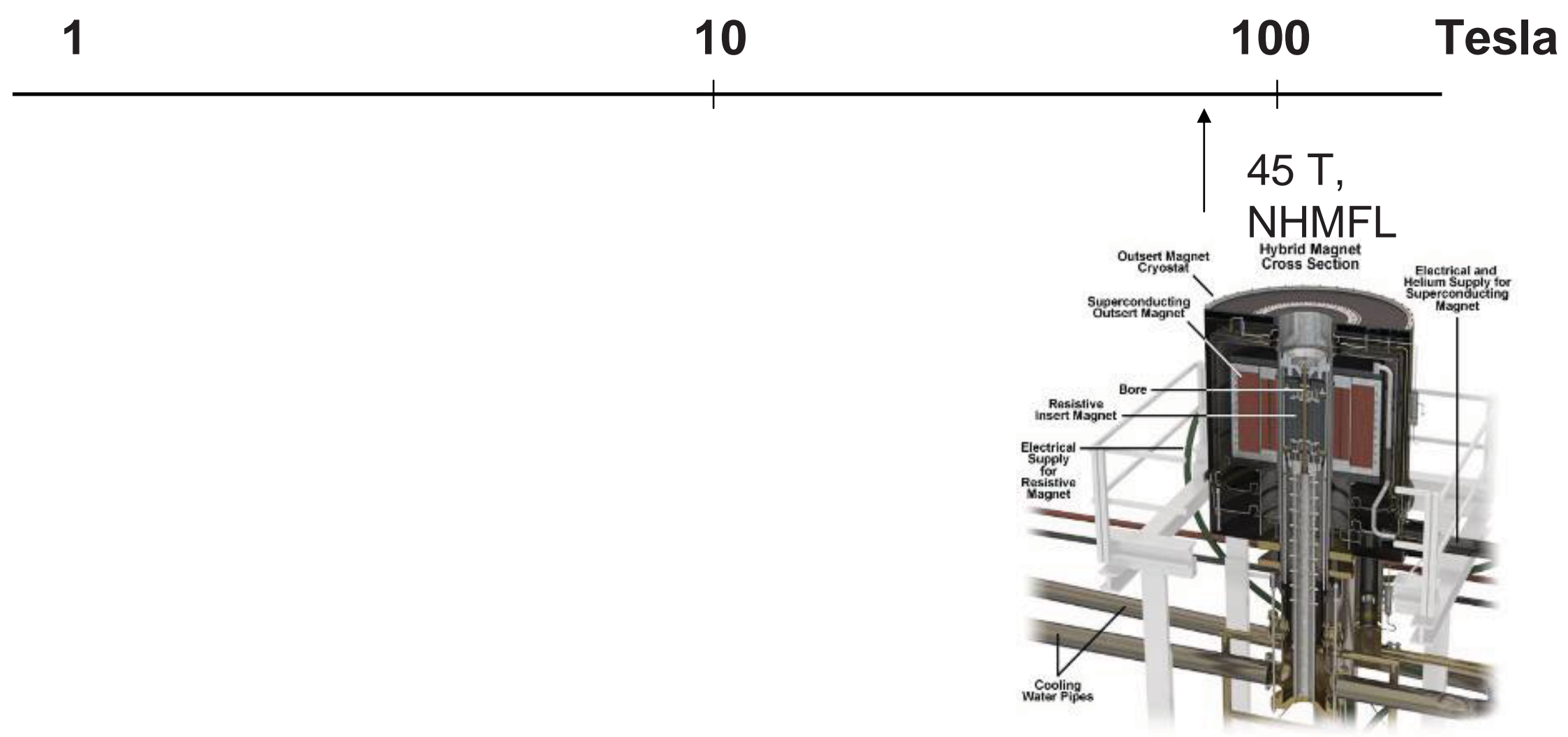


DC field installation LNCMI Grenoble

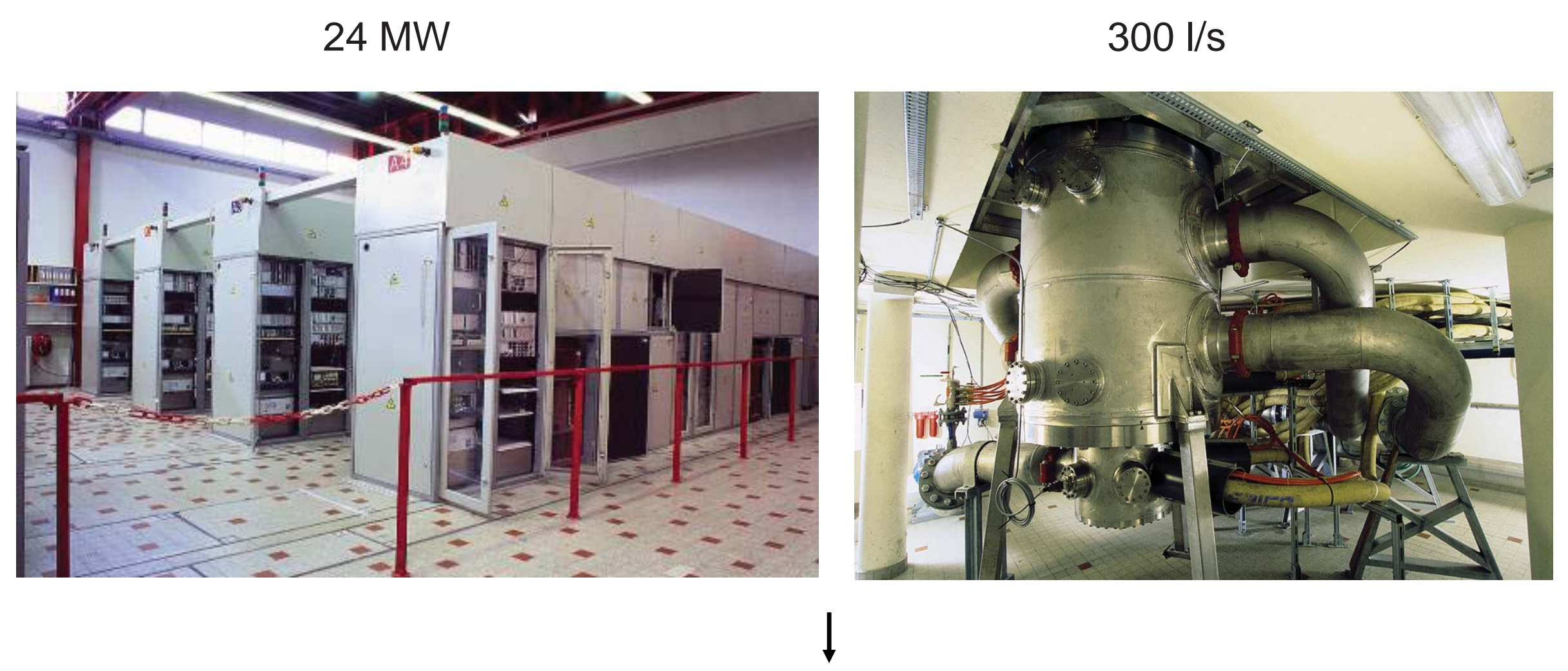

35 Tesla

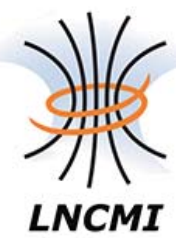


State of the art resistive magnet technology
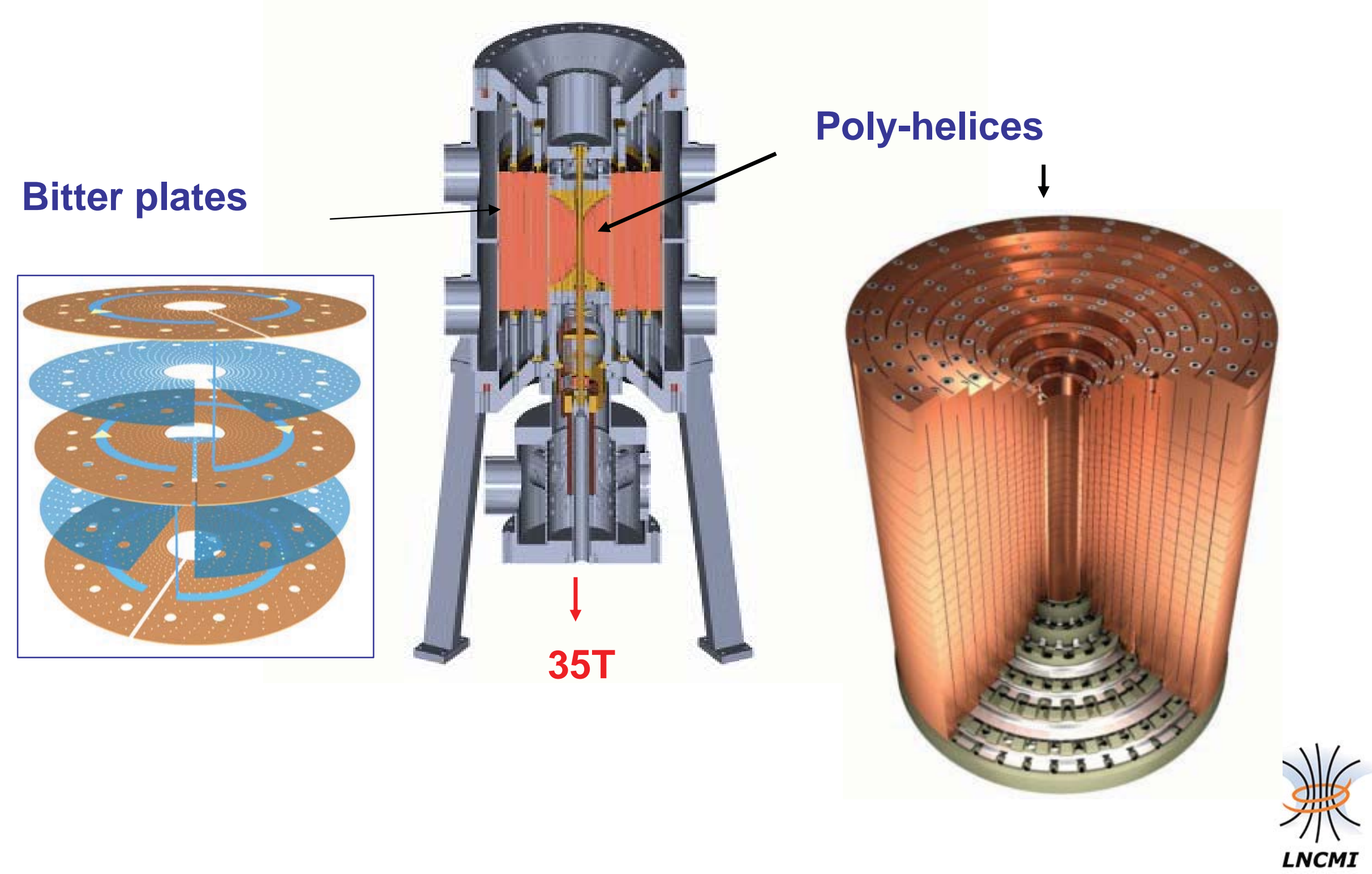


\section{Pulsed magnetic fields}

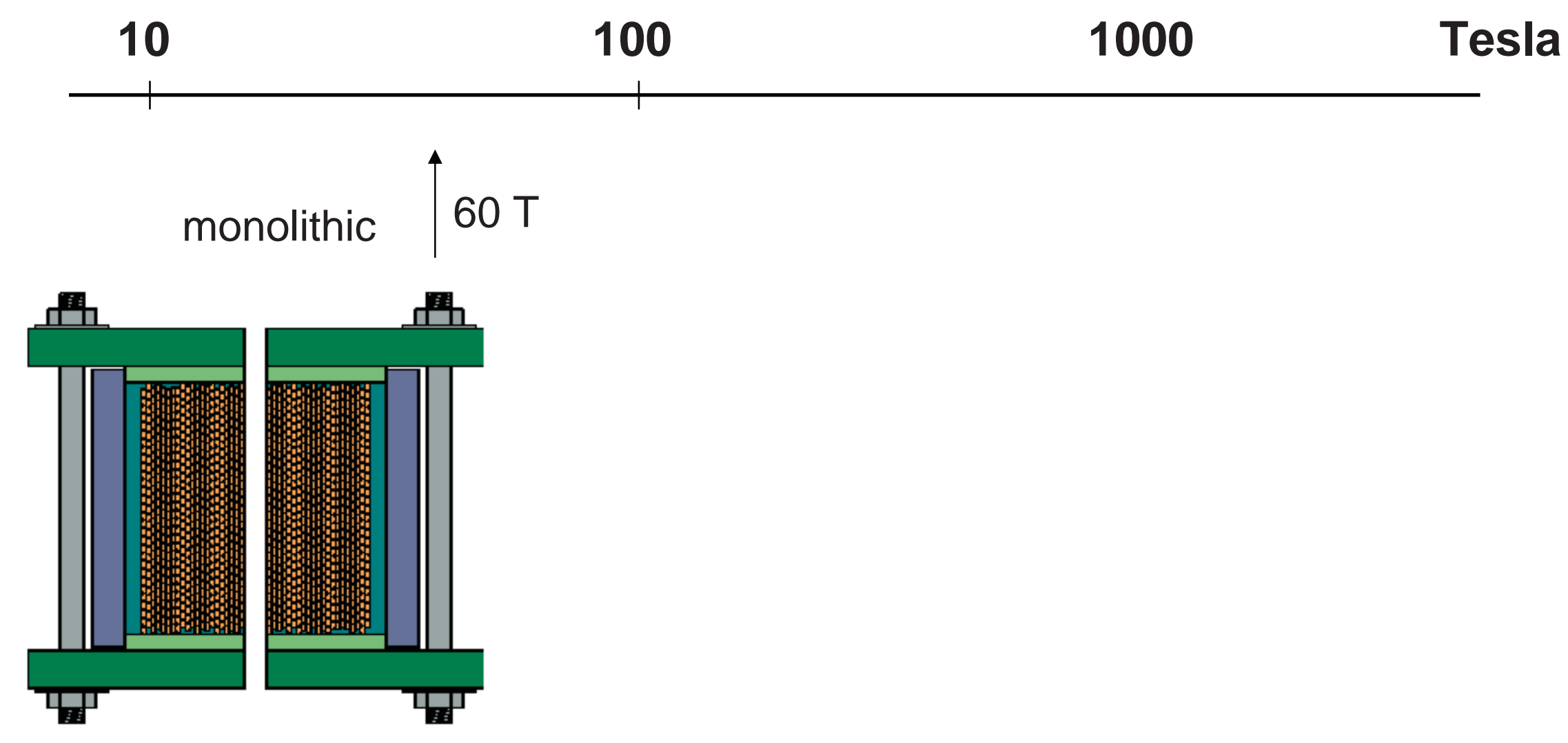




\section{Pulsed magnetic fields}

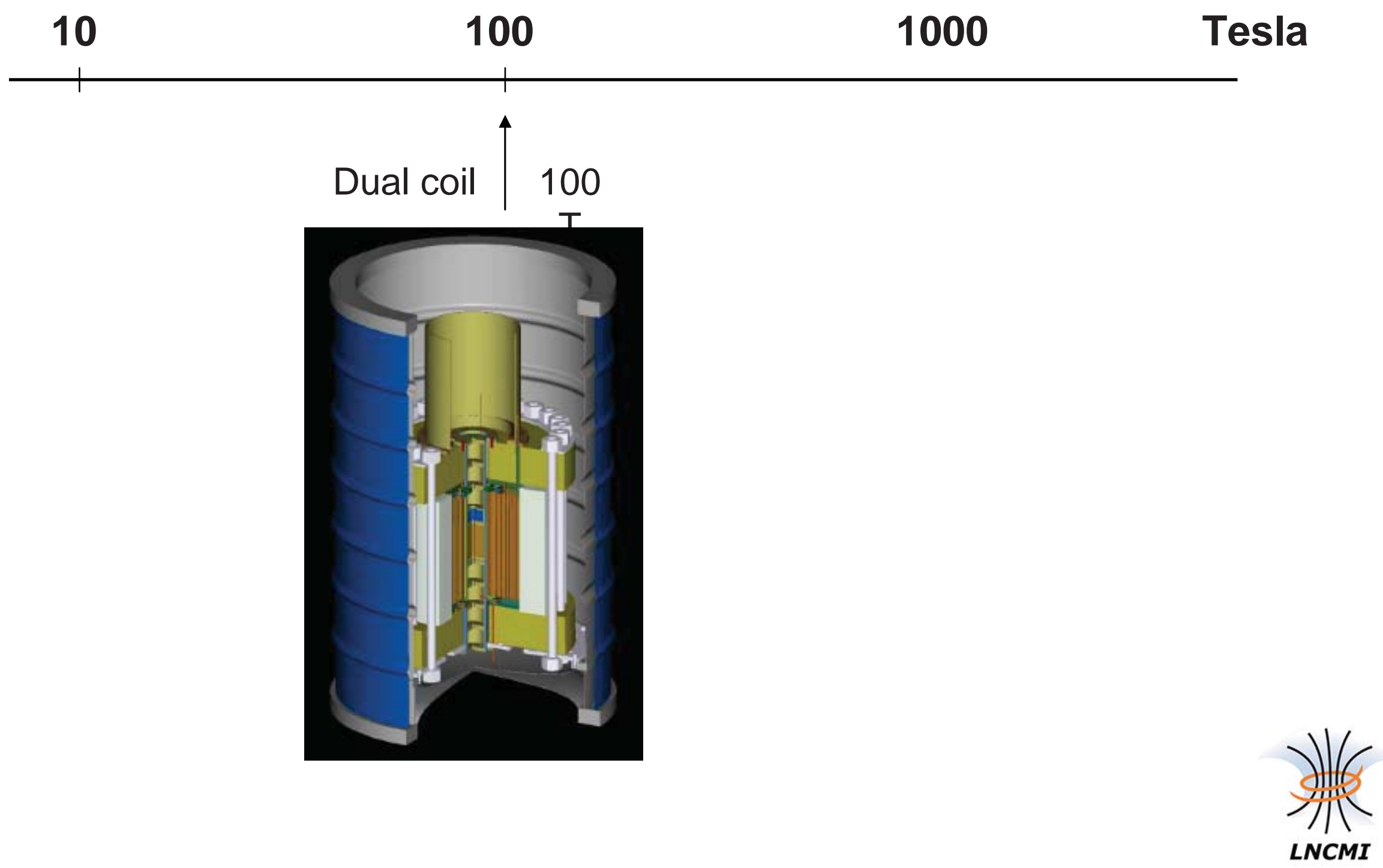




\section{Pulsed magnetic fields}

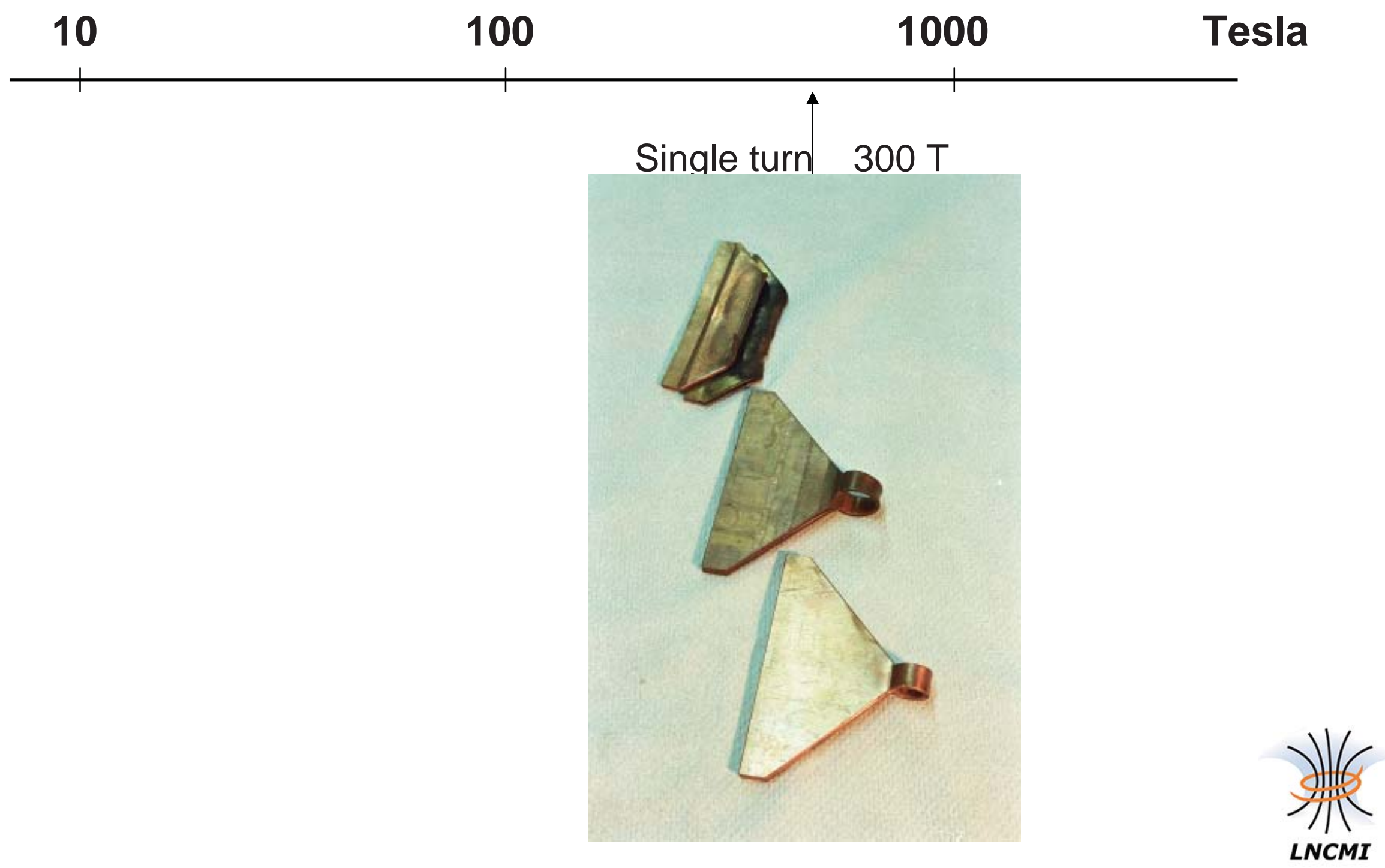




\section{Pulsed magnetic fields}

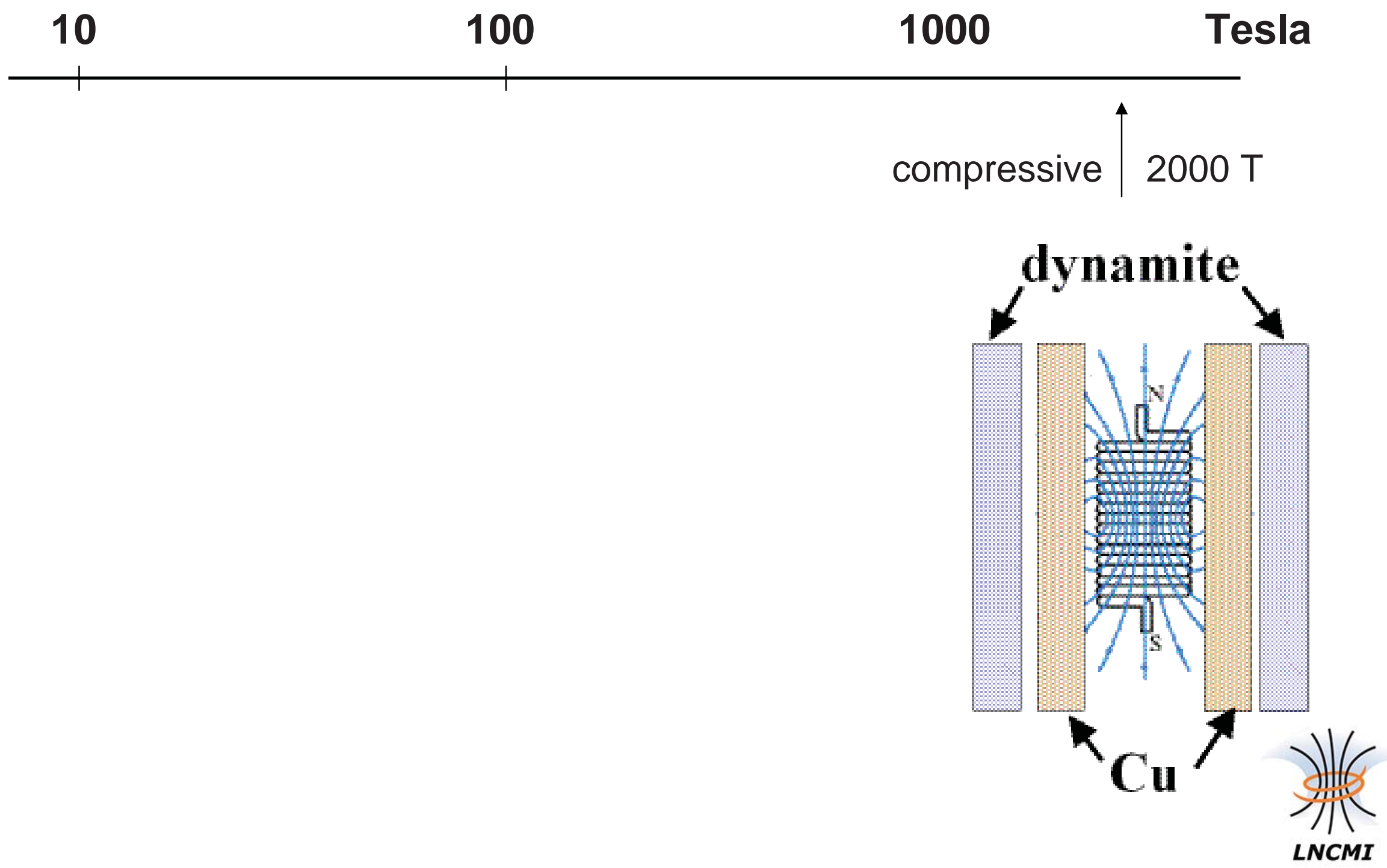




\section{Pulsed magnetic fields}

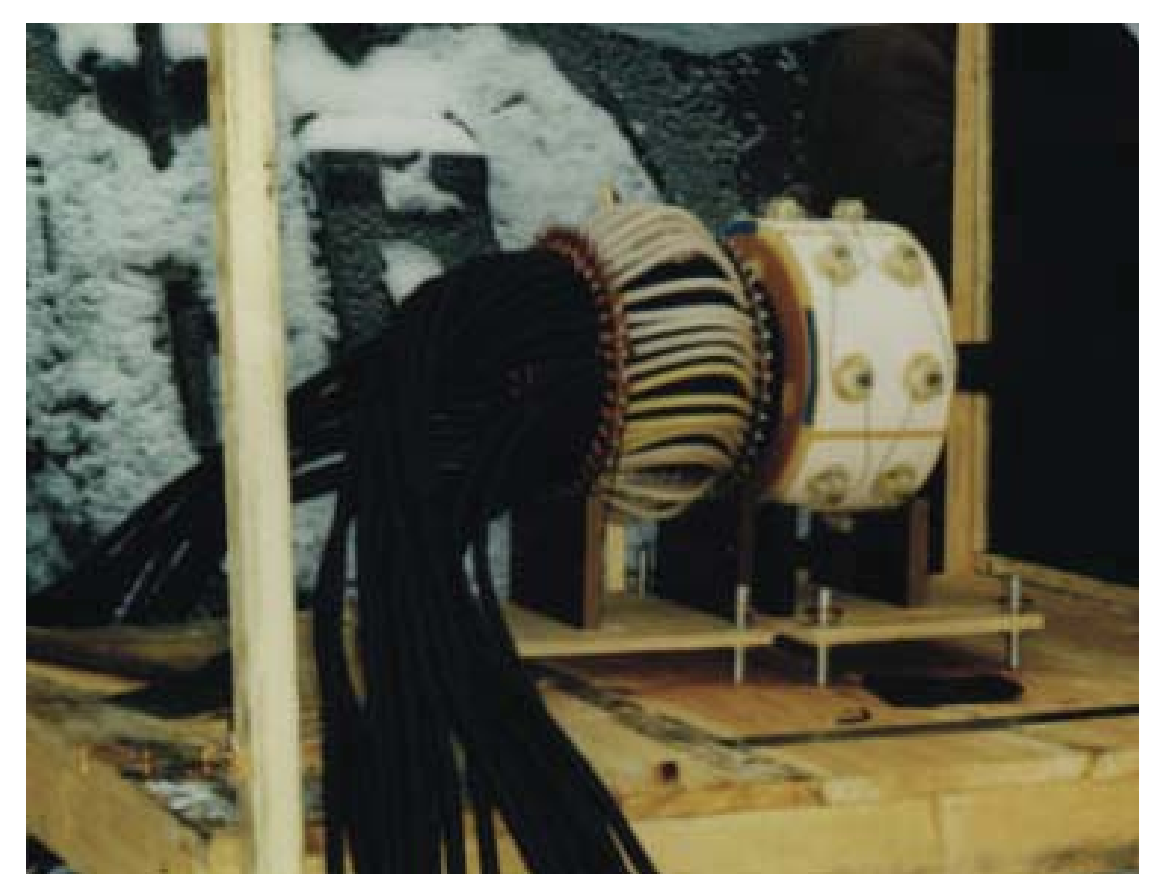




\section{Pulsed magnetic fields}

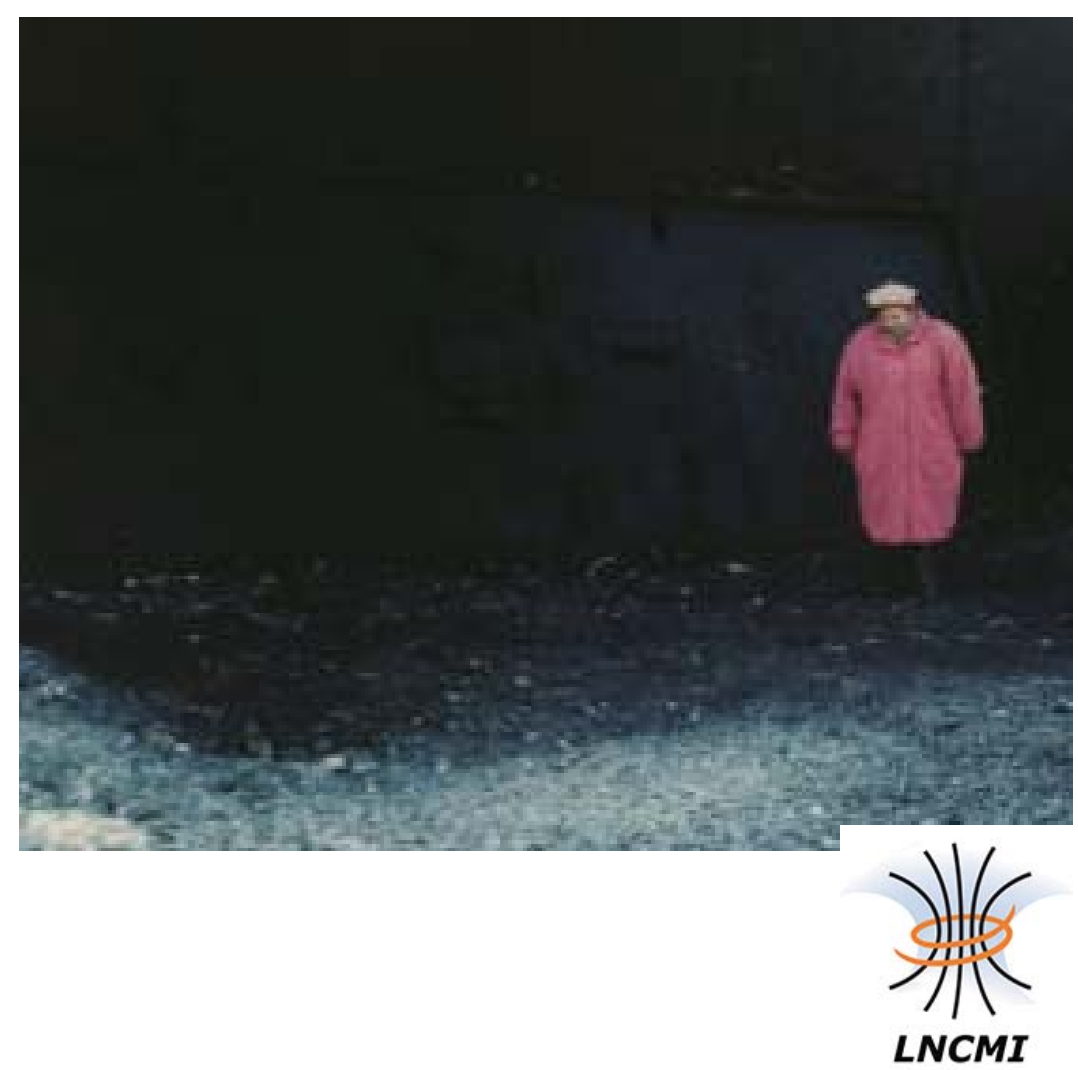




\section{Pulsed field installation LNCMI Toulouse}

14 MJ, 24 kV, 1 GW; 80 Tesla

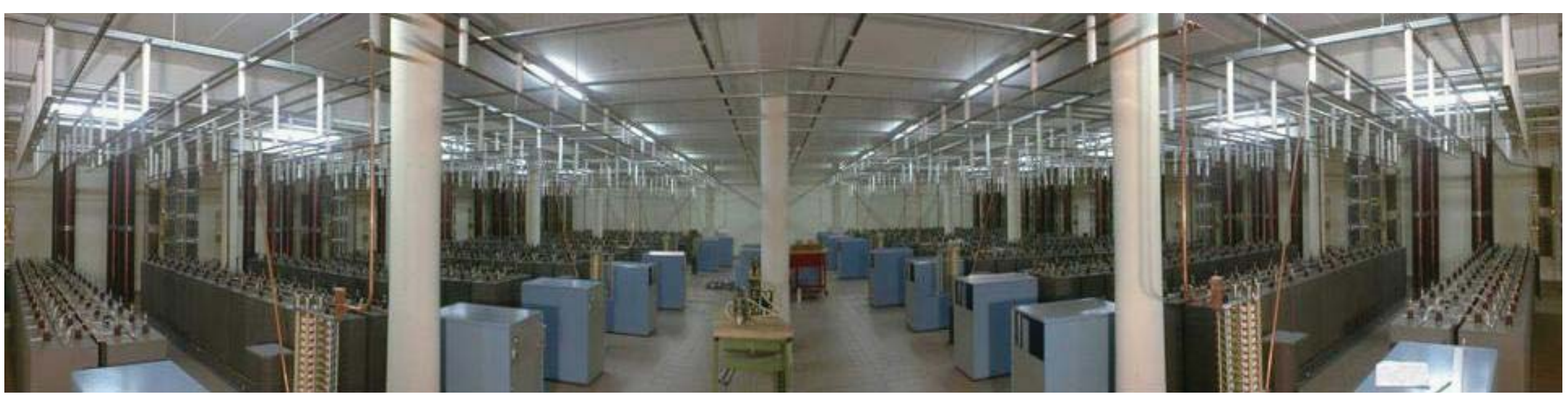

$1 \mathrm{GW}$ peak power

0,2 MW average power

$\downarrow$

$\lim _{11}(6$

Electrical power is not an issue!

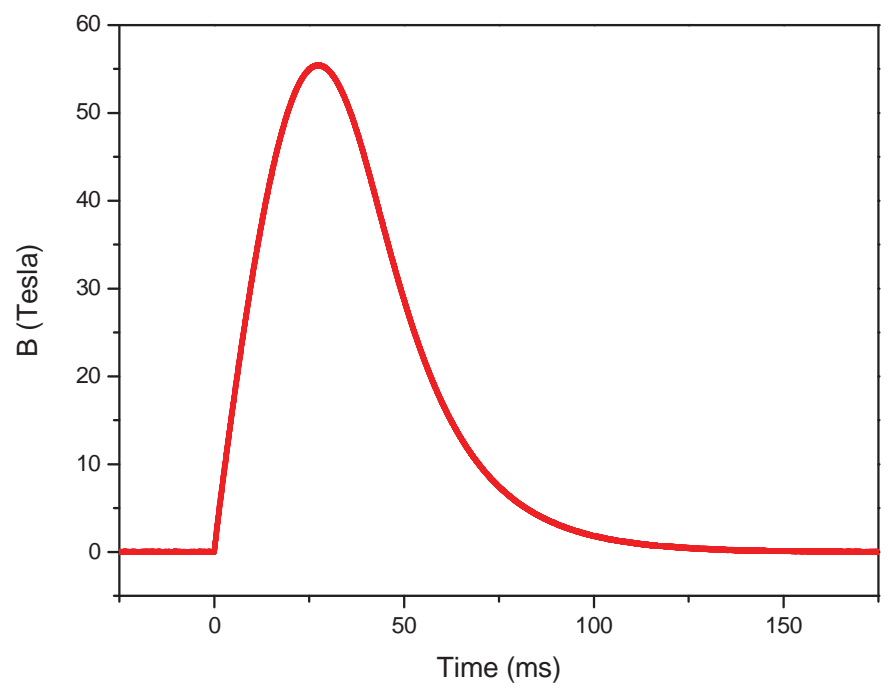


HLD: the world's largest capacitor bank : 50 MJ, 5 GW

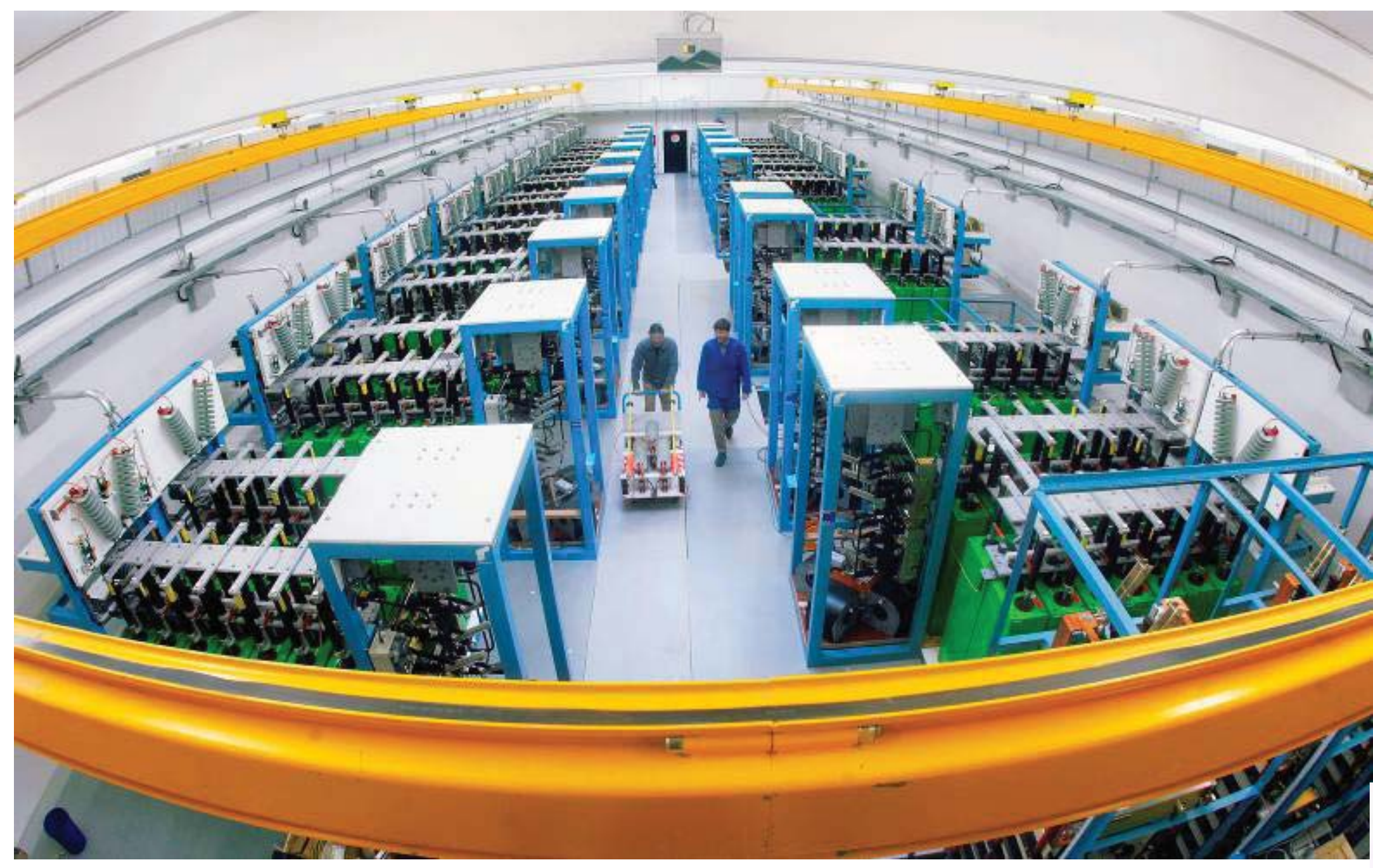




\section{Semi-destructive fields; installation MegaGauss}
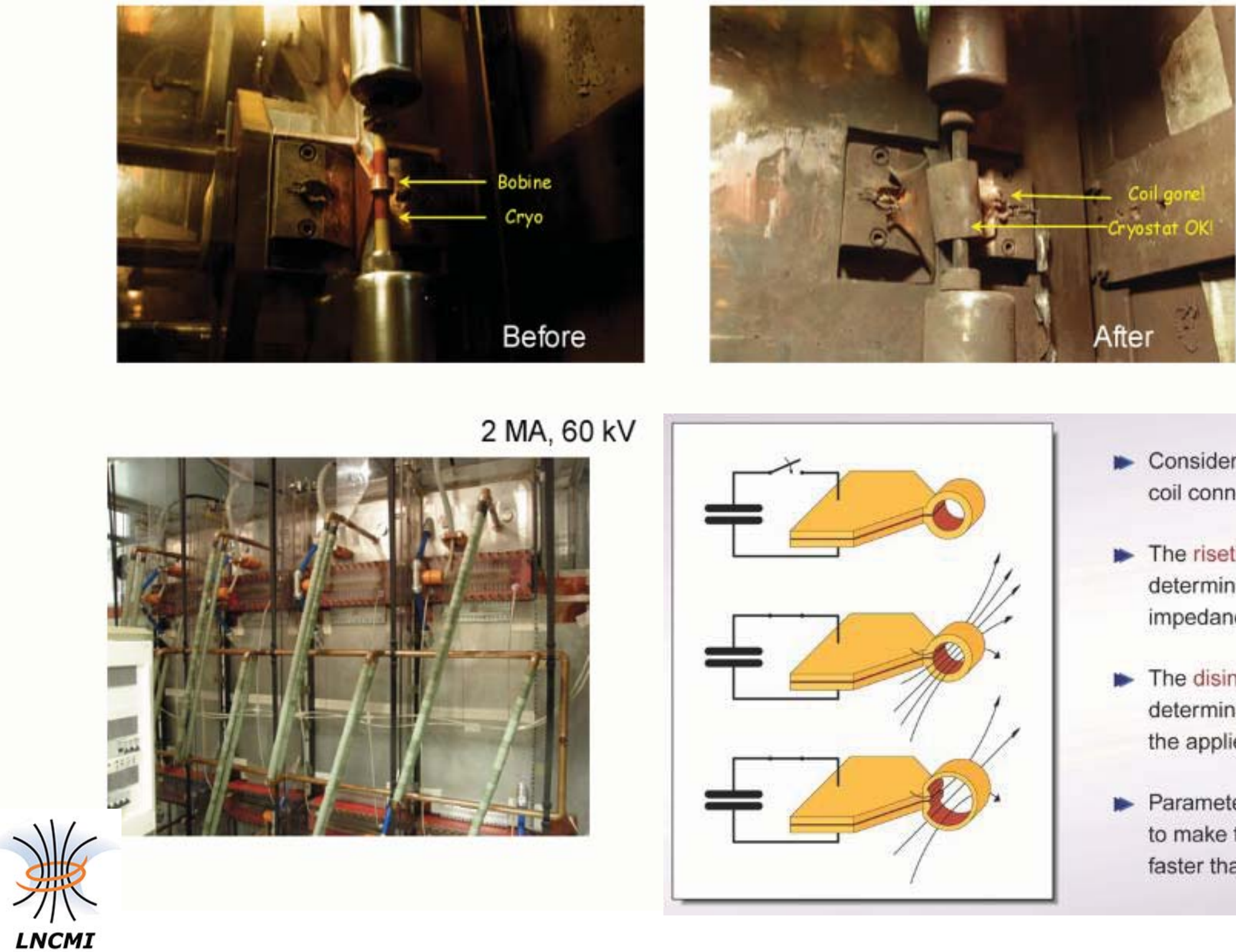

- Consider a simple disposable coil connected to a capacitor

- The risetime of the field is determined by the circuit impedance and capacitance

- The disintegration of the coil is determined by its mass and the applied force

- Parameters can be adjusted to make the field risetime faster than the disintegration 
Optical experiments in Megagauss

Faraday rotation of a CdS cristal up to 165 Tesla

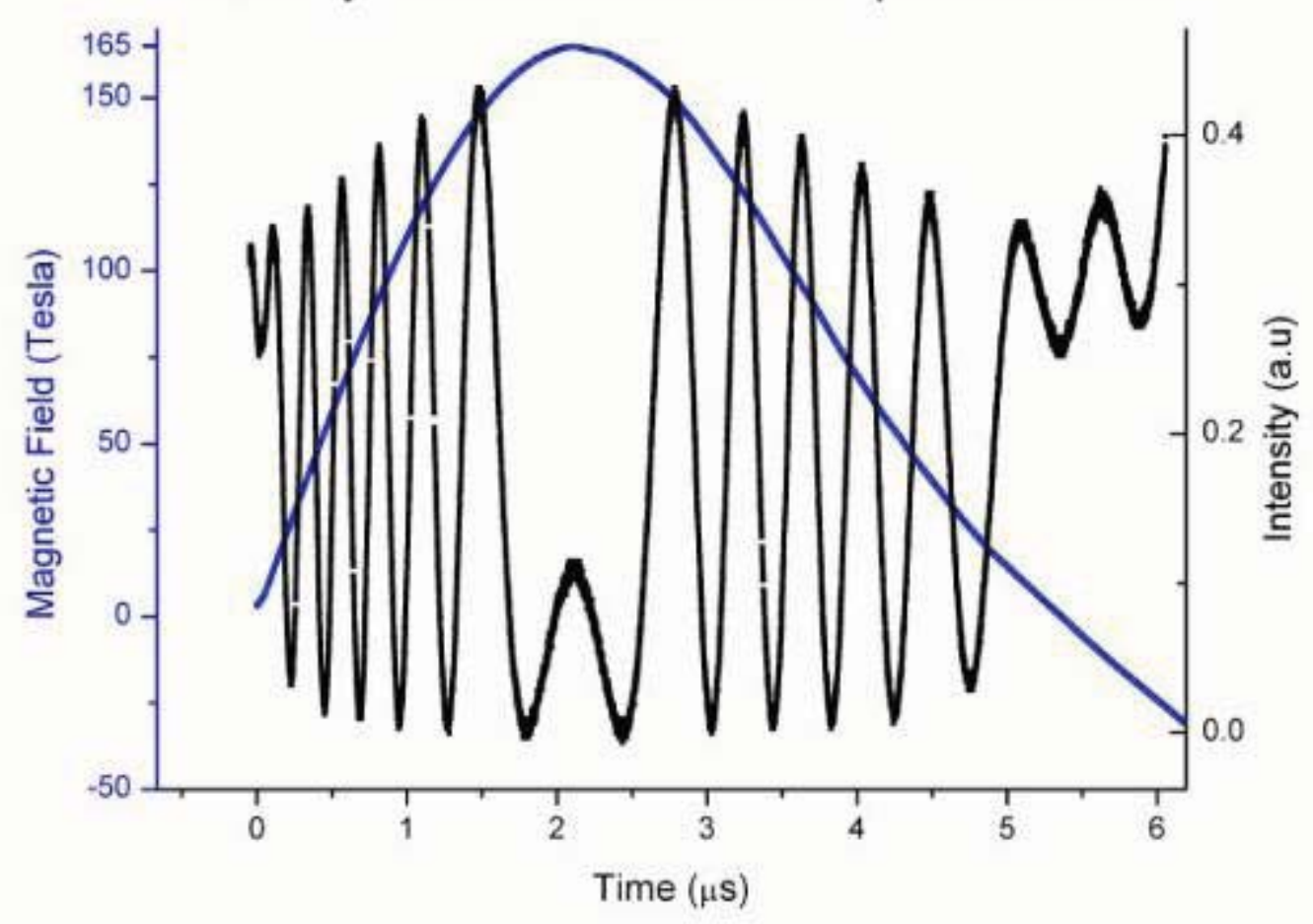

Current limit: $200 \mathrm{~T}$, potentially up to $300 \mathrm{~T}$ 


\section{Mobile pulsed field installation}

\section{LNCMI}

The small average power and absence of heavy cooling installations make mobile pulsed high field installations possible:

High field $X$ ray scattering at ESRF (180 kJ, 16 kV, 30 Tesla)

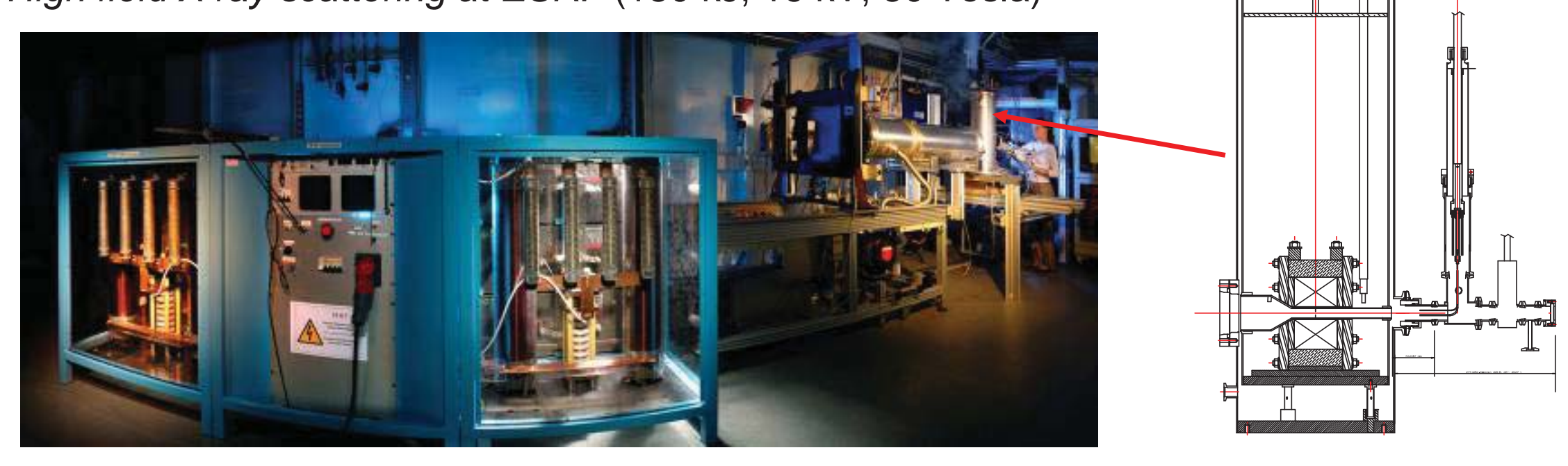

'Light shining through wall', LULI

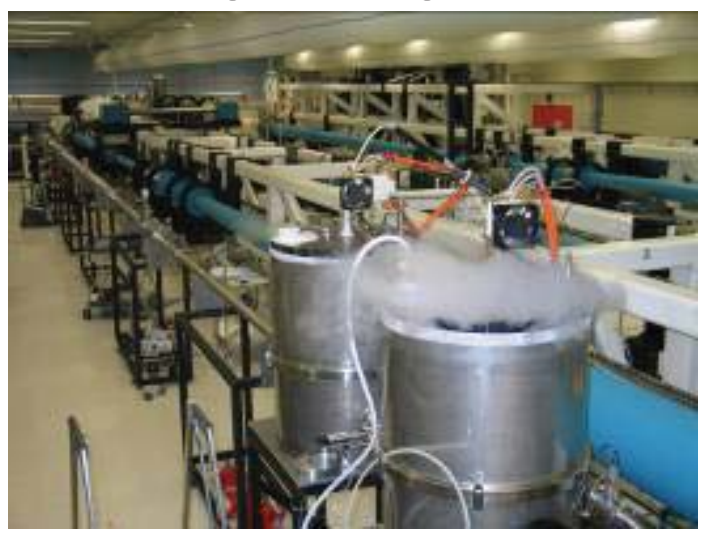

'X ray scattering' ESRF

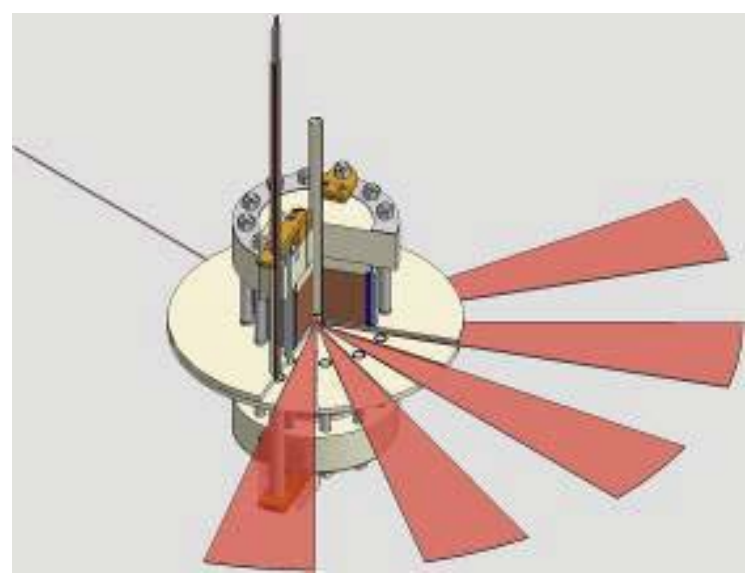


Laser induced plasma in a high magnetic field at LULI
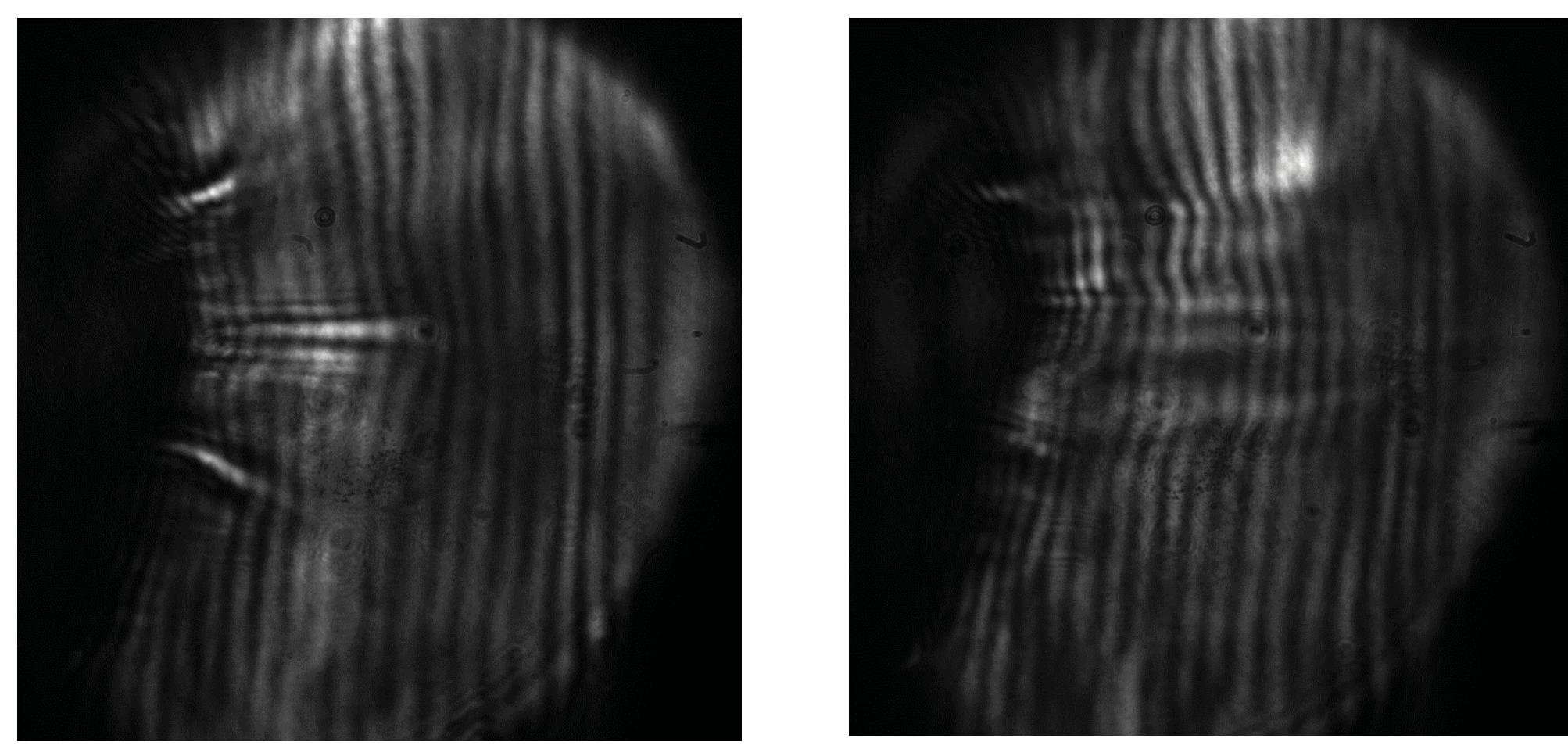

$\mathrm{B}=0$

$$
B=20 \mathrm{~T} \longrightarrow
$$

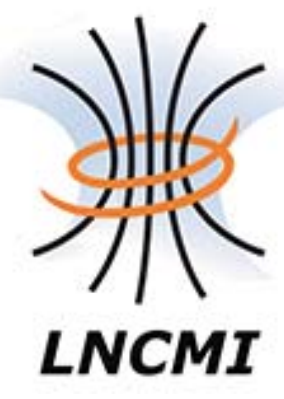




\section{Second generation mobile installation:}

Elastic neutron scattering in pulsed magnetic fields

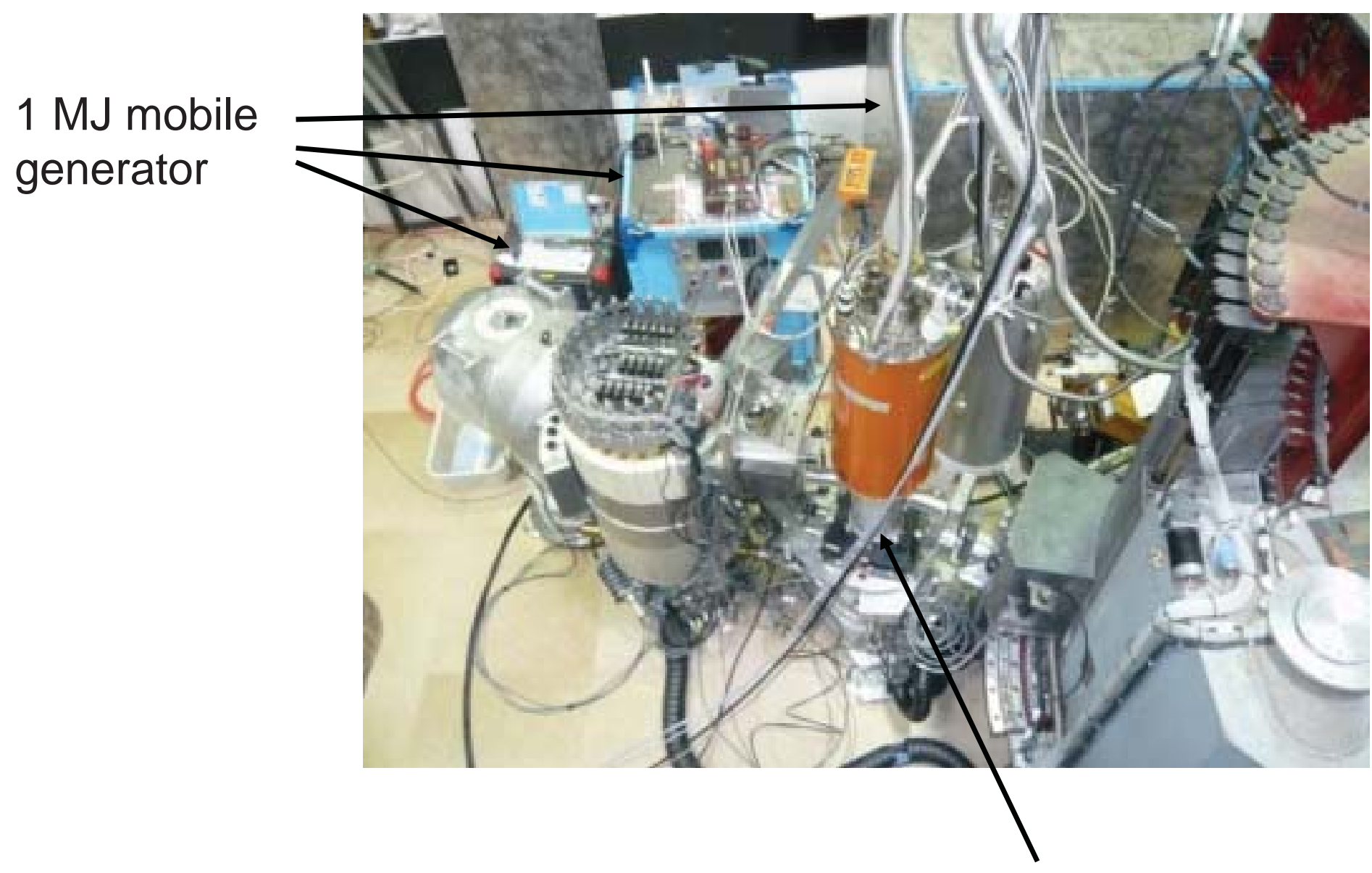

IN22 at ILL

40 T magnet 
Next generation mobile pulsed field installation

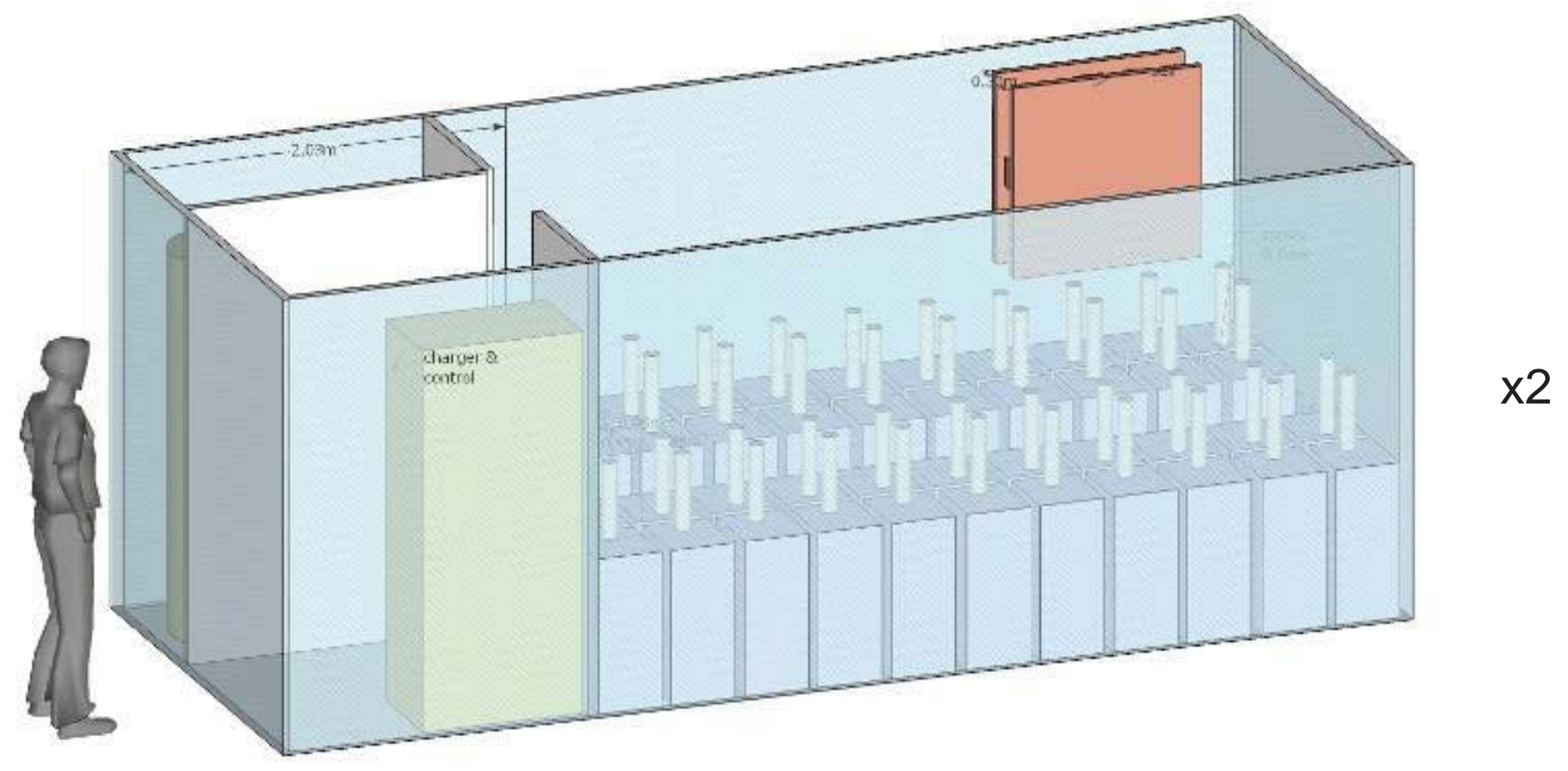

$6 \mathrm{MJ}, 24 \mathrm{kV}$ in $2 \times 20$ foot sea containers $\rightarrow 80 \mathrm{~T}$

(under construction, available Feb. 2013)

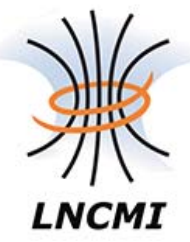




\section{Future developments in high magnetic field generation}

High $T_{\mathrm{C}}$ superconductors

Materials research

Improved cooling techniques

New magnet designs 


\section{State of the art technical superconductors}

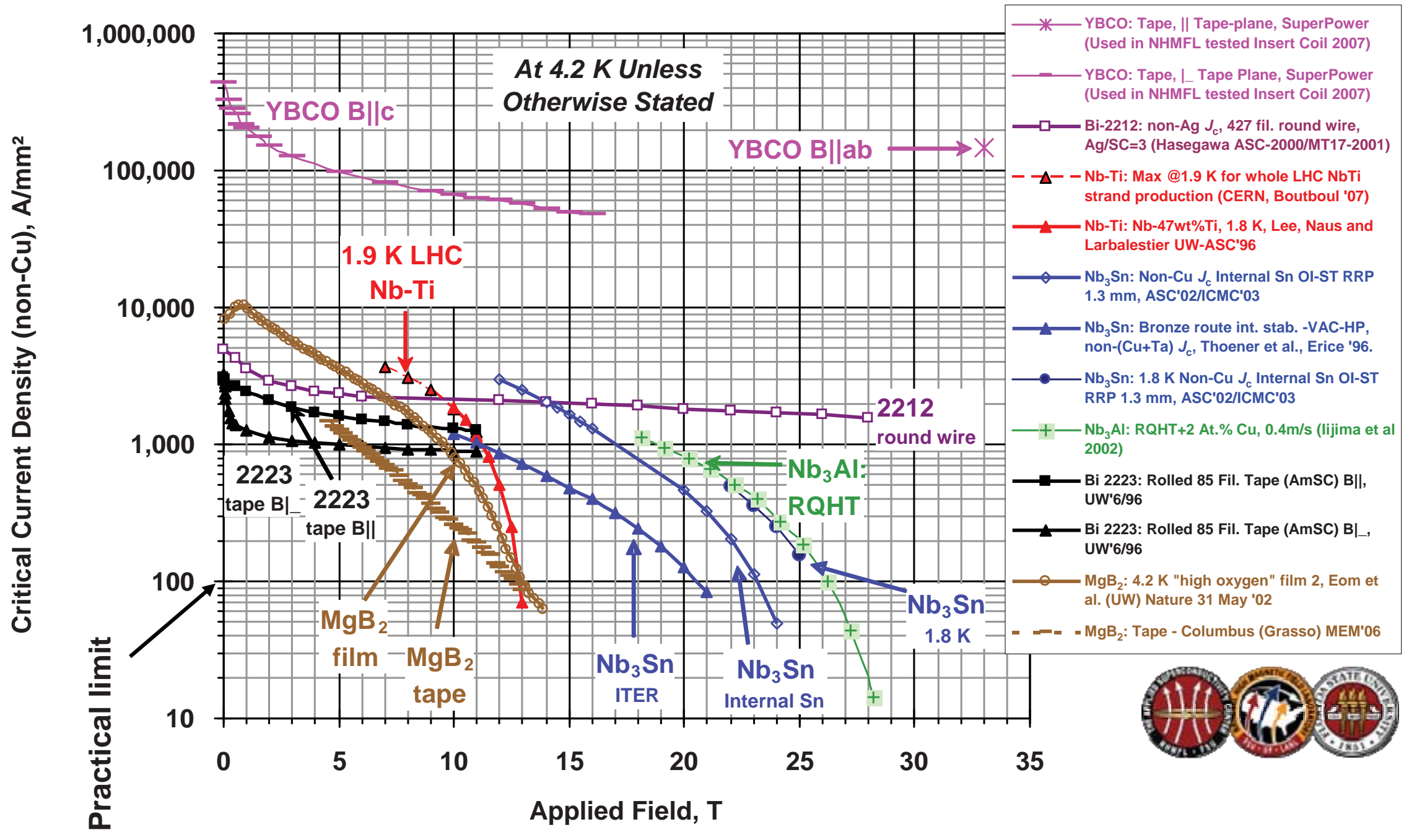


State of the art high $\mathrm{T}_{\mathrm{c}}$ superconductor tape

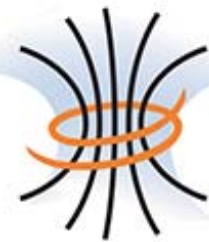

LNCMI

Ac(0) Versus Stress at $77 \mathrm{~K}$

Tspe DD \#M3-393-1-ES504-569t4

- Phenomenal $\mathrm{J}_{\mathrm{c}}$ in the YBCO - $20 \times 10^{6}$ $\mathrm{A} / \mathrm{cm}^{2}$ at $25 \mathrm{~T}$

- YBCO is $\sim 1 \%$ of cross-section

- $50 \%$ is high strength superalloy

- Well suited for high field solenoids
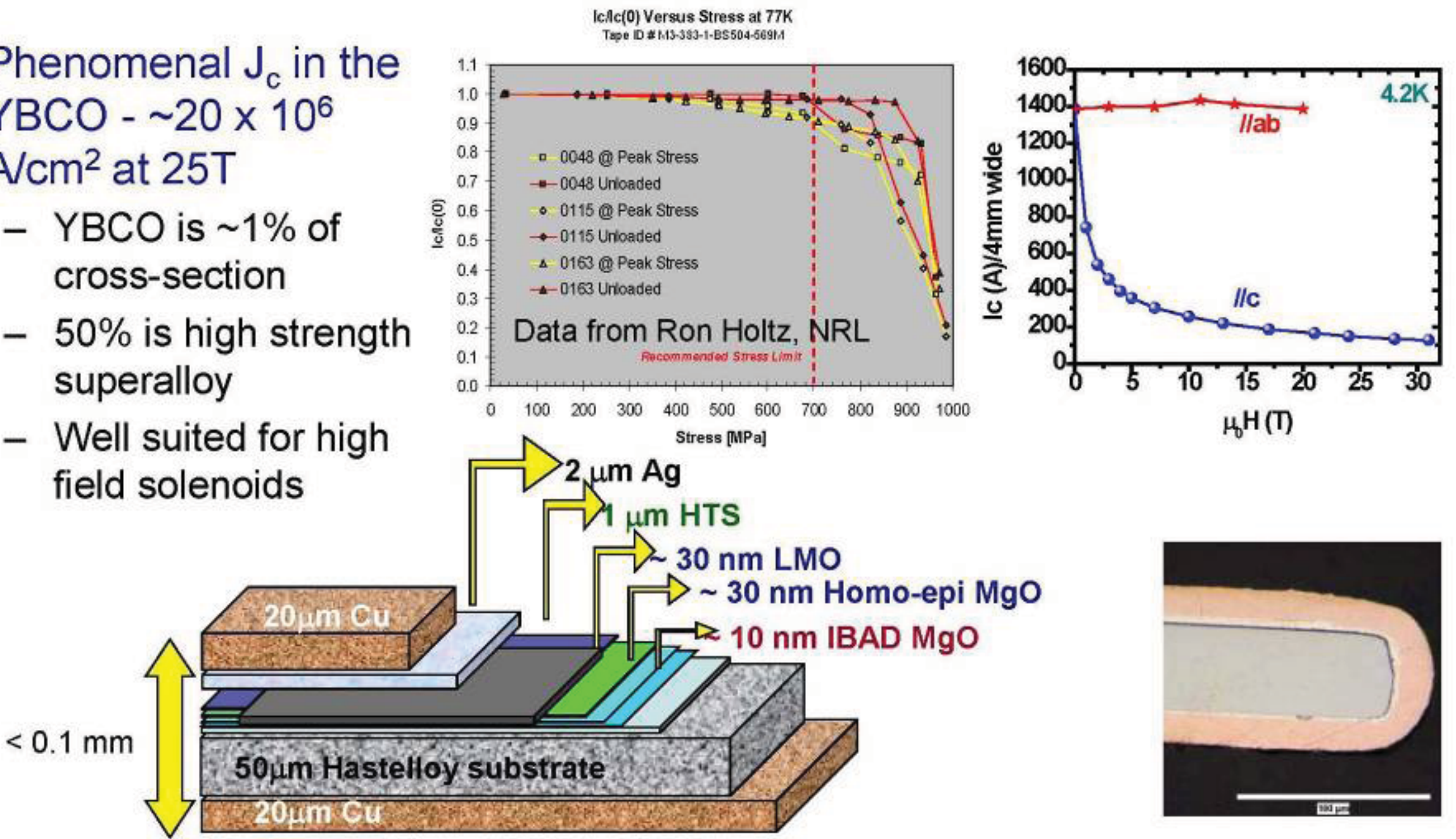
First high $T_{c}$ superconductor magnet, under construction

Progress $\mathrm{HT}_{\mathrm{c}}$ insert coils

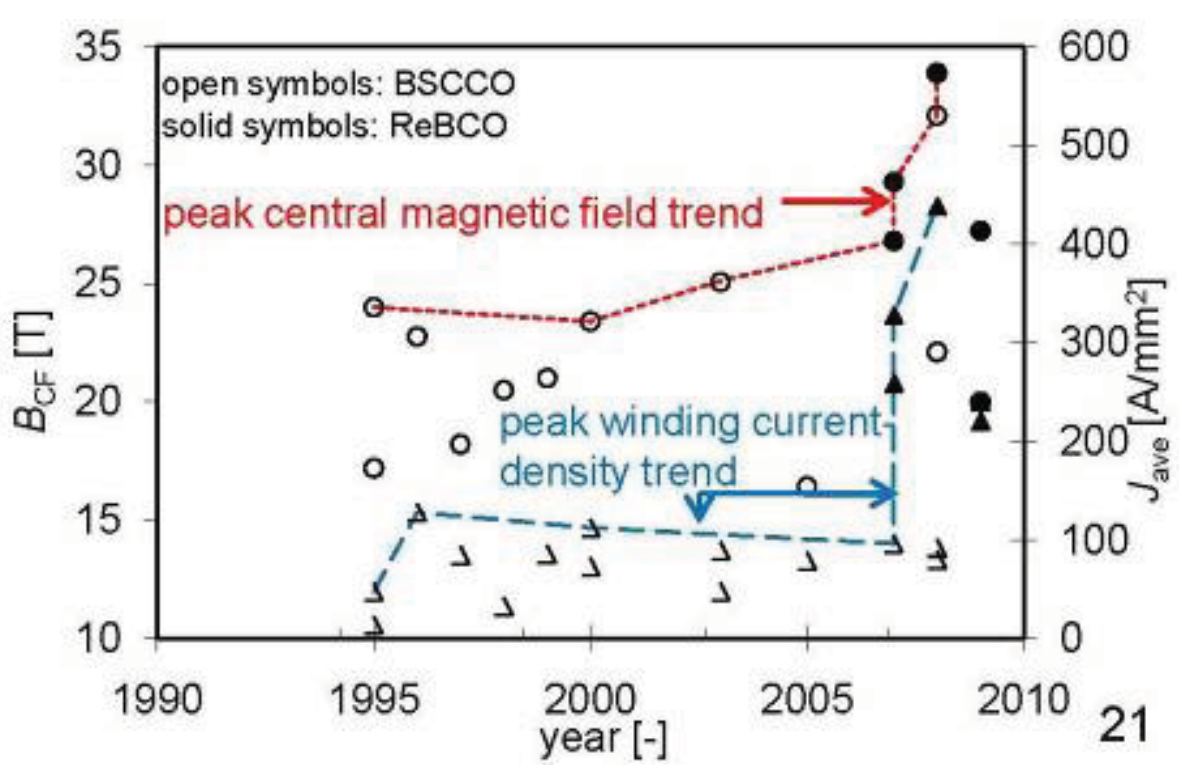

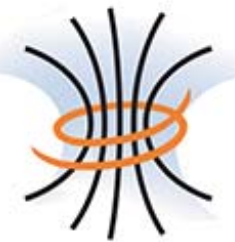

LNCMI

NHMFL $32 \mathrm{~T} \mathrm{HT}_{\mathrm{C}}$ under construction

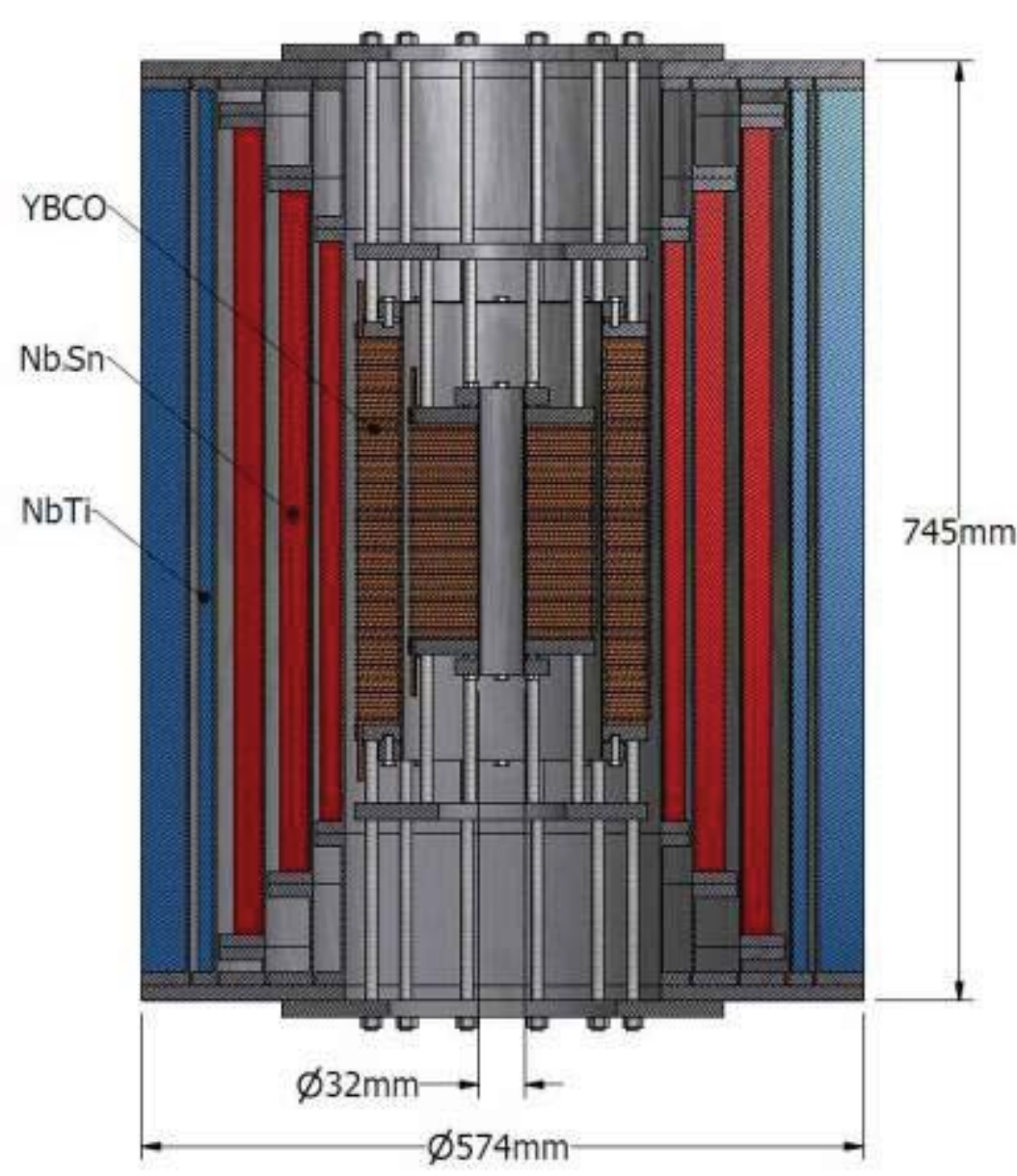

$4 \mathrm{M} €$, 4 years development 


\section{Materials research for resistive magnet technology}

Trade-off between mechanical resistance and electrical conductivity

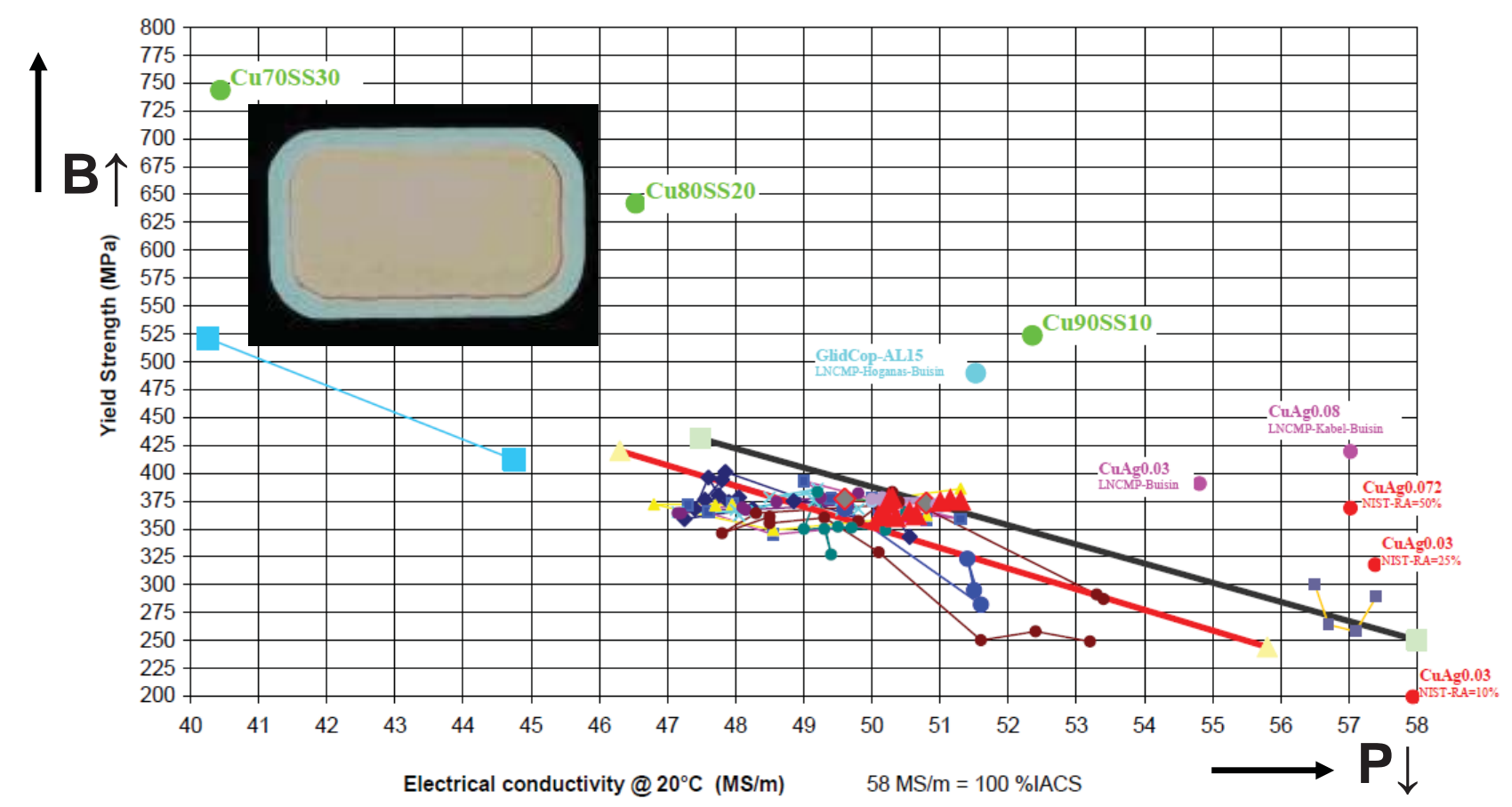

Extensive and very specific materials R\&D necessary to make progress

Sub-industrial quantities needed; too big for labs, too small for industry 
Dedicated coil designs for scattering experiments
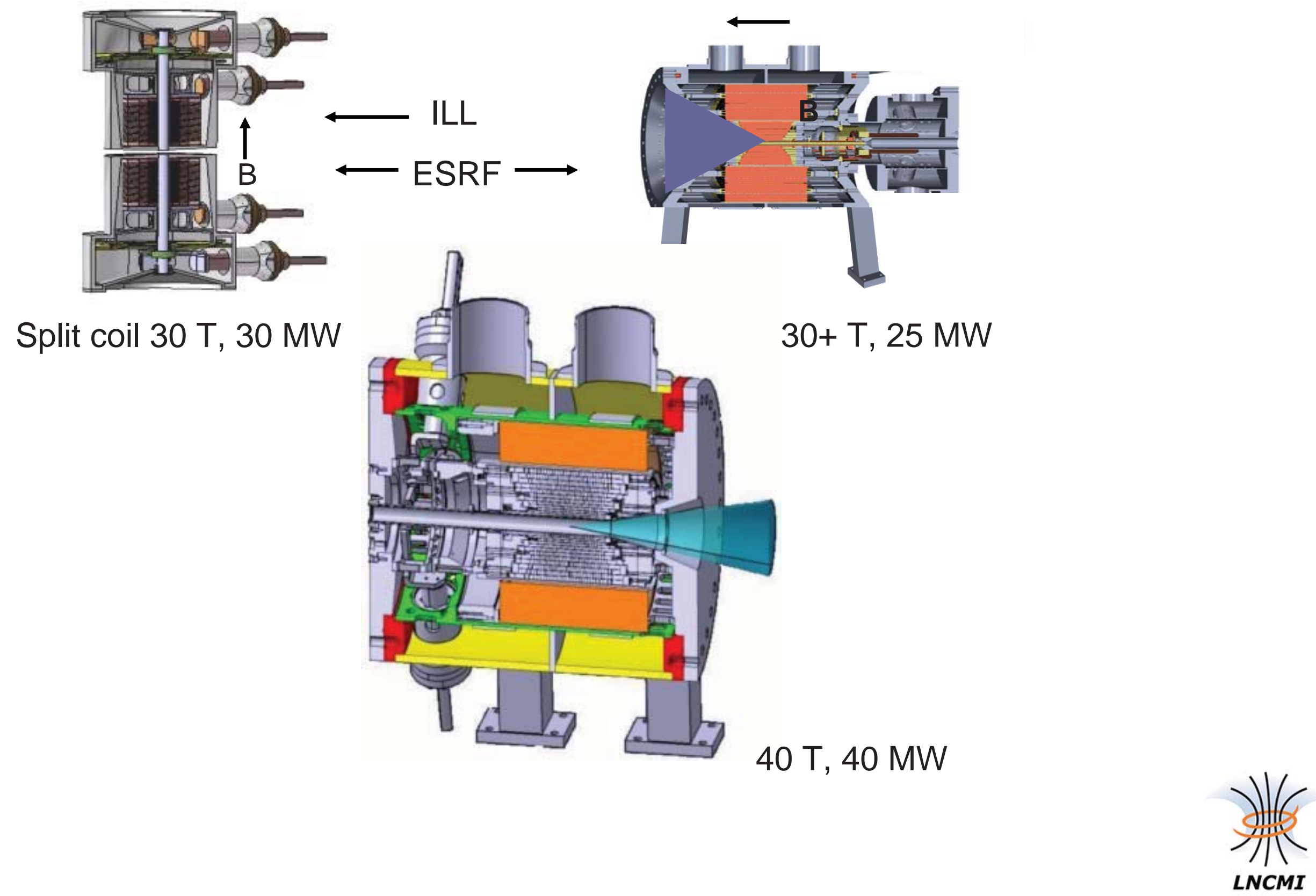


\section{Hybrid magnets}

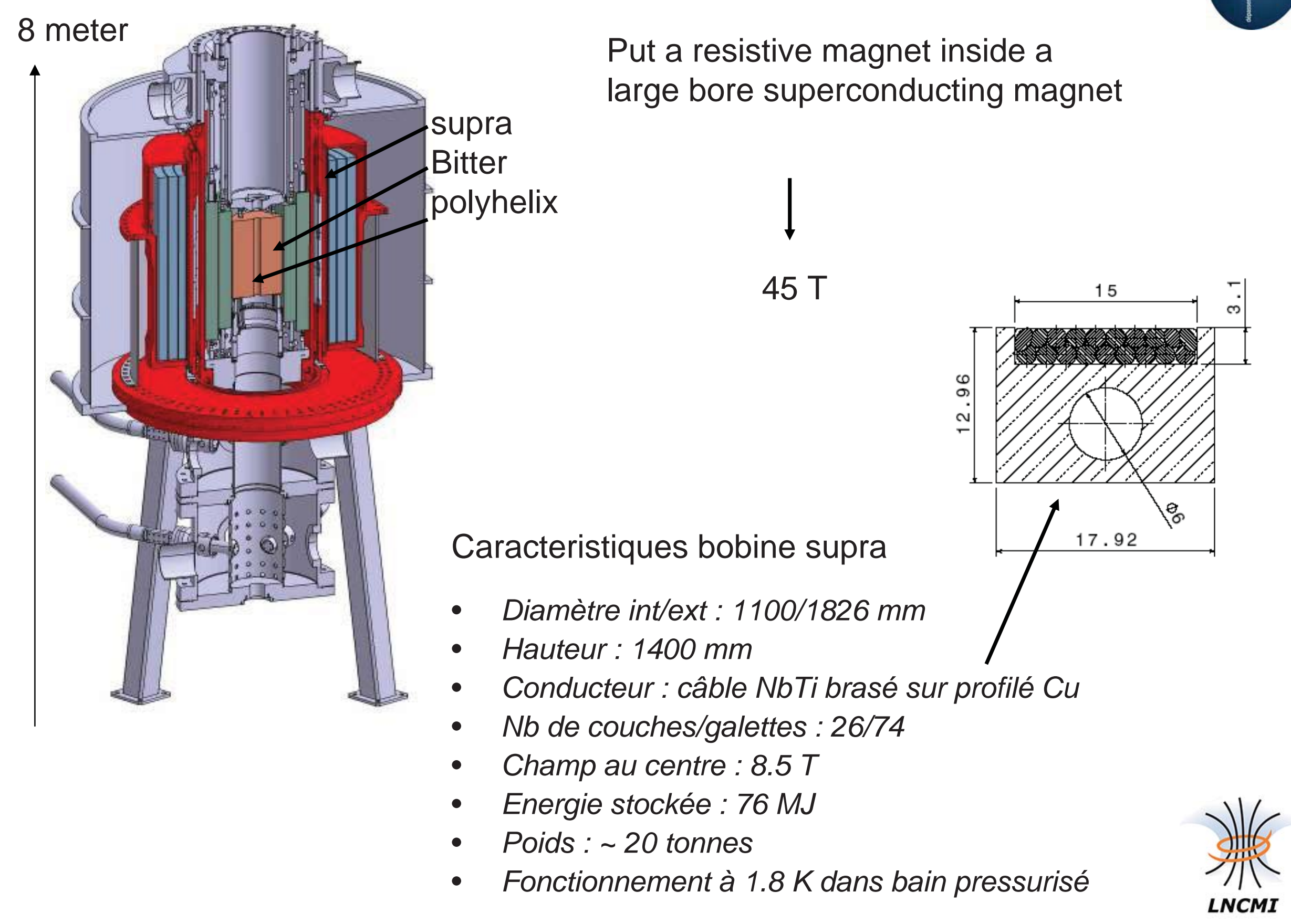




\section{Ultrarapid cooling of pulsed magnets: Cu-inox polyhelix}
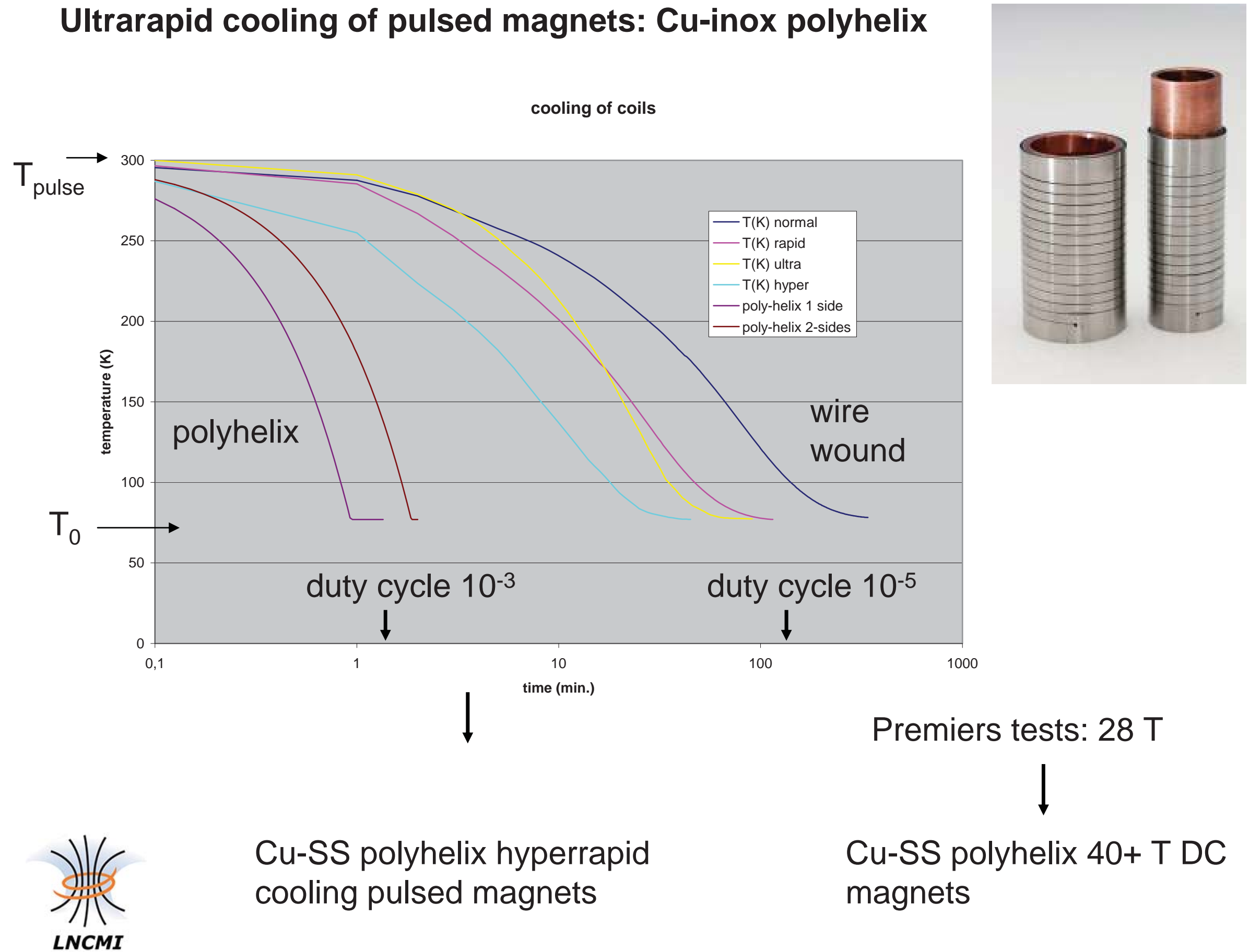

Cu-SS polyhelix hyperrapid cooling pulsed magnets
Cu-SS polyhelix 40+ T DC magnets 


\title{
High magnetic field facilties
}

\author{
Existing facilities
}

Use of high field facilities

European Magnetic Field Laboratory 


\section{International context ; Europe}

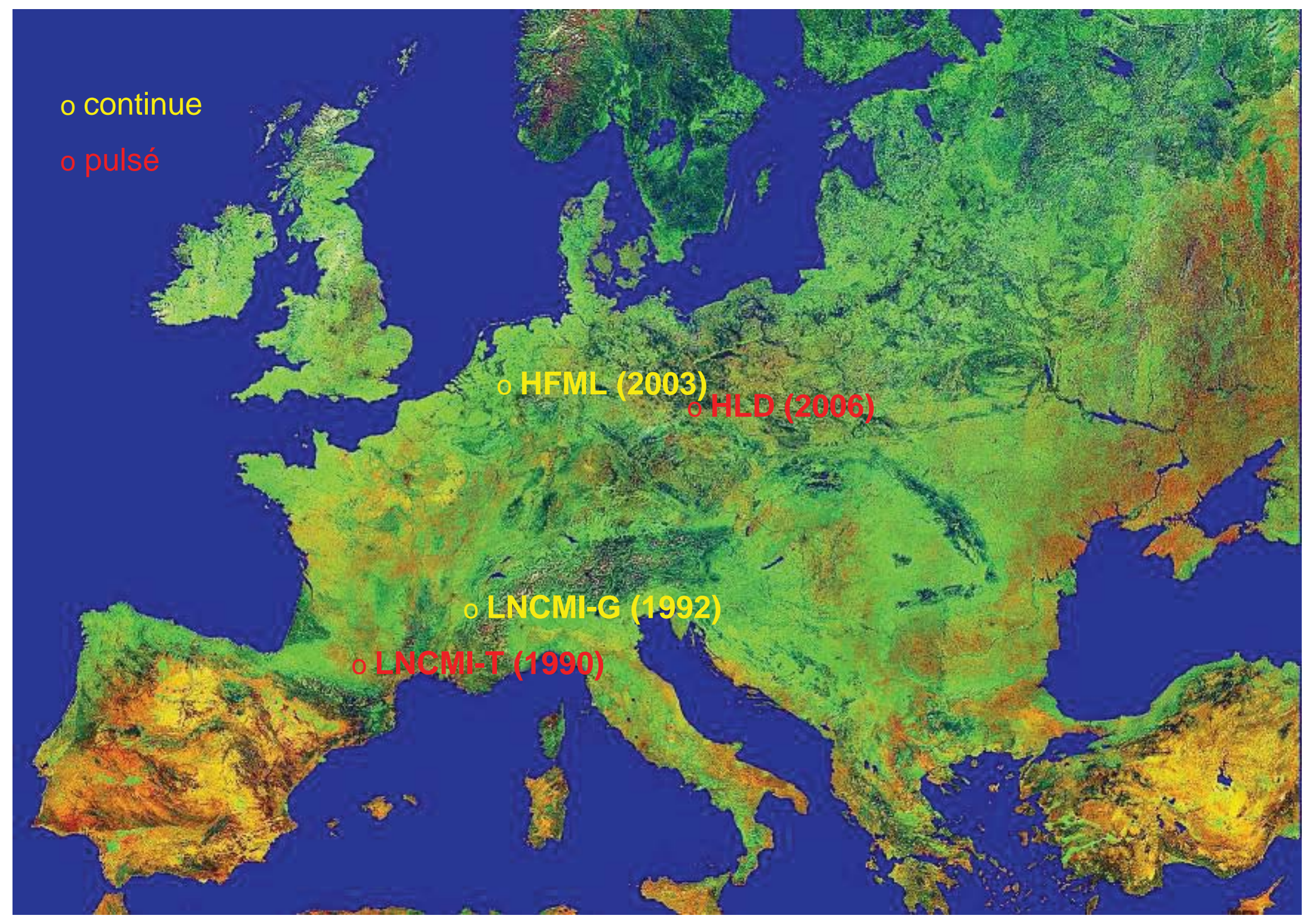




\section{HLD Dresden}

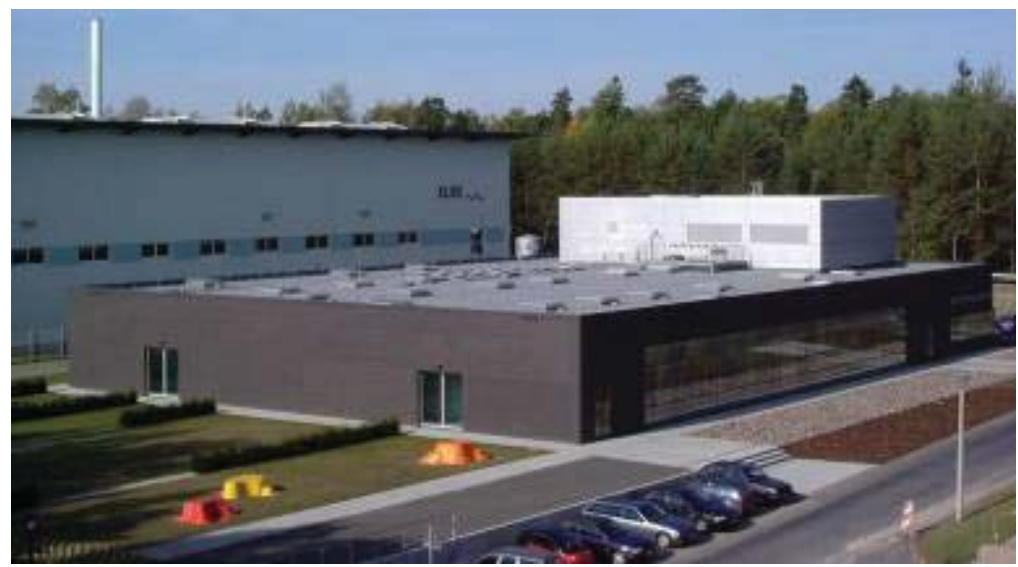

LNCMI Toulouse

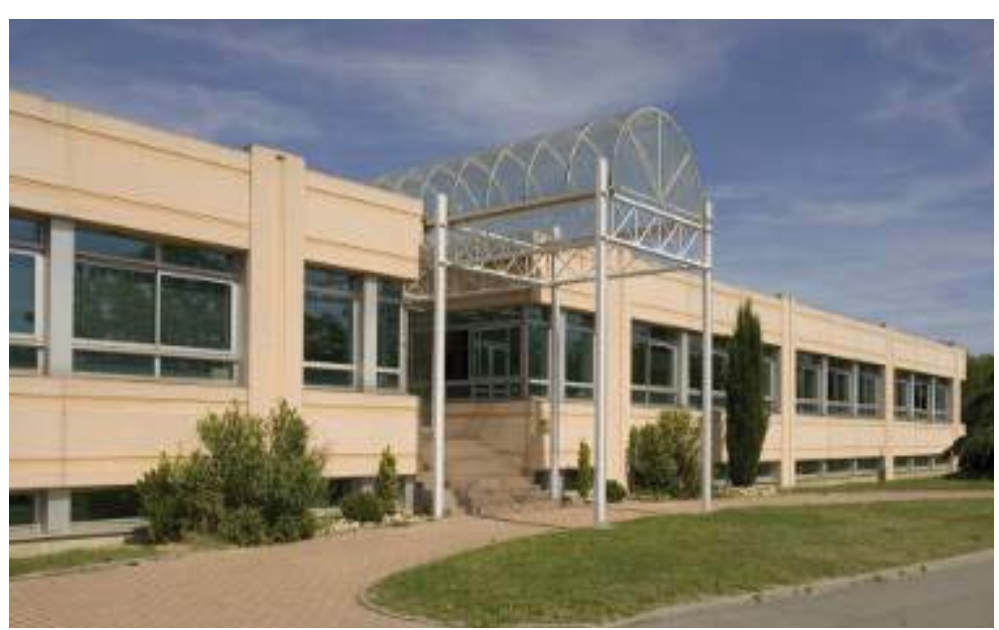

HFML Nijmegen

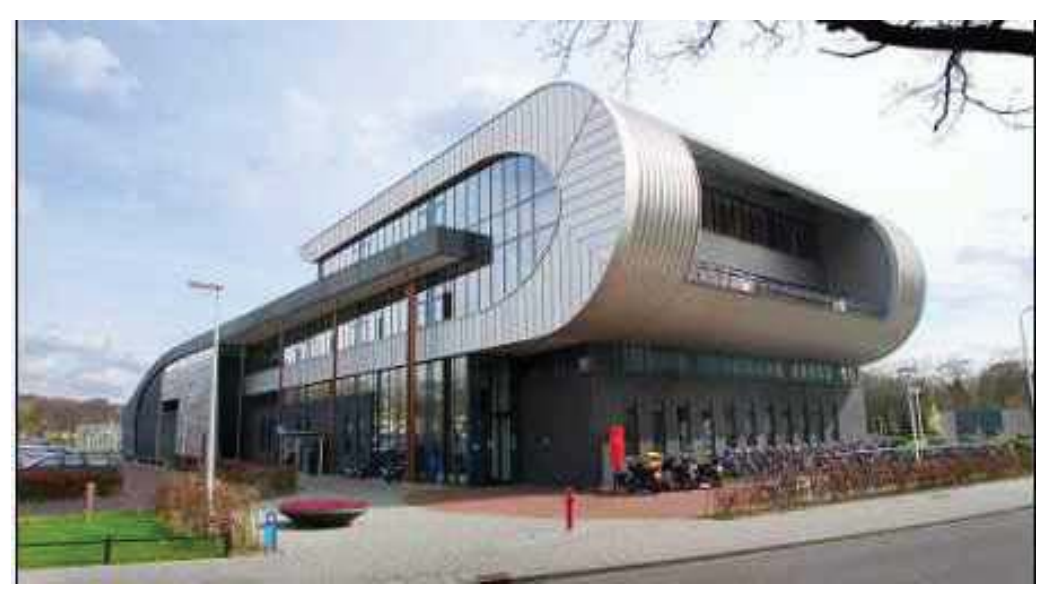

LNCMI Grenoble

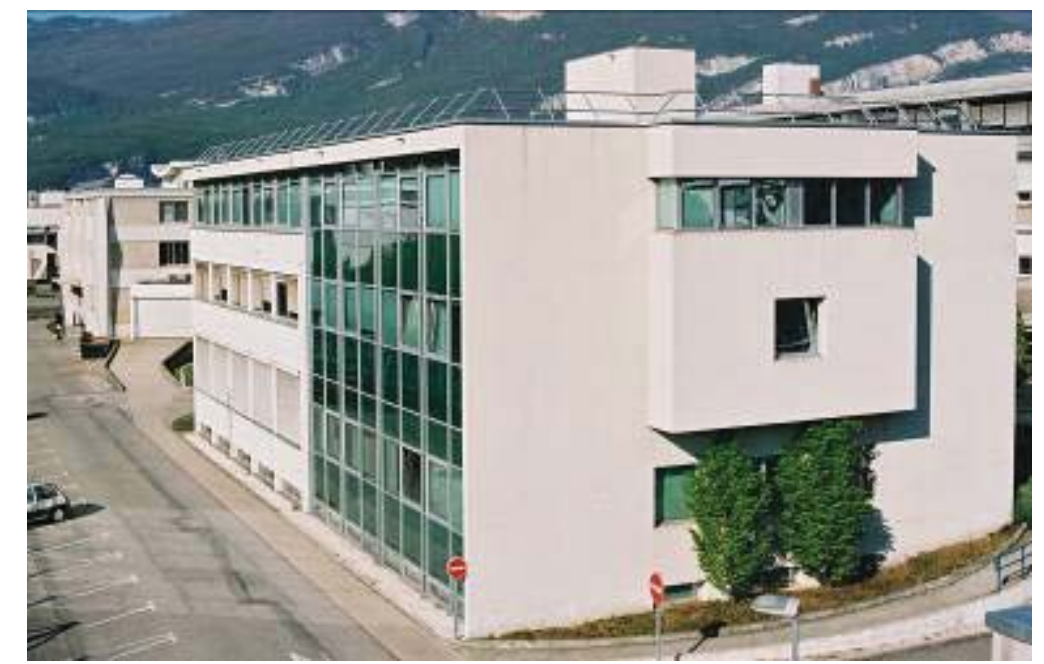




\section{International contexte: outside Europe}
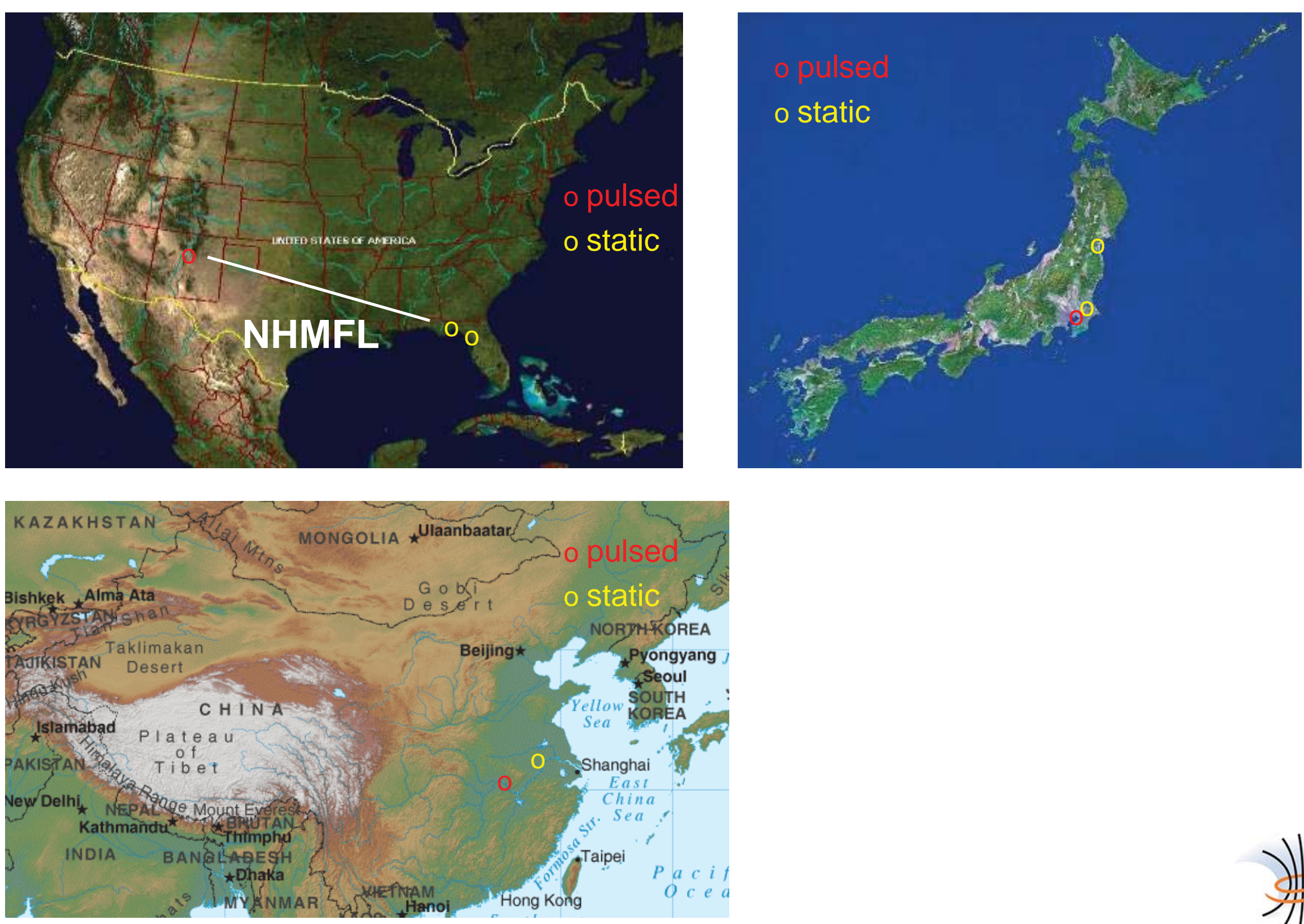


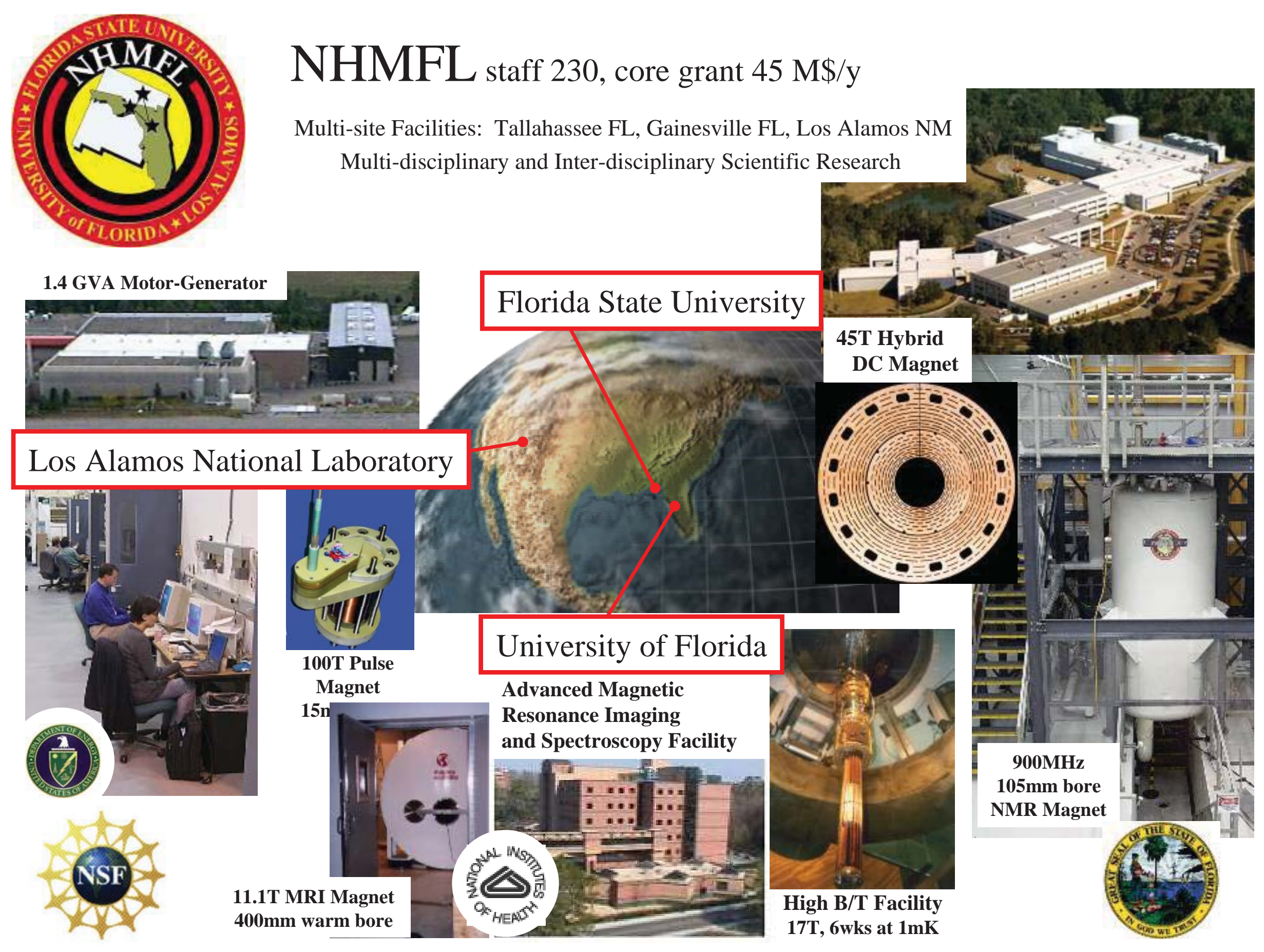


New high field facility in Heifei (China), 26 M€

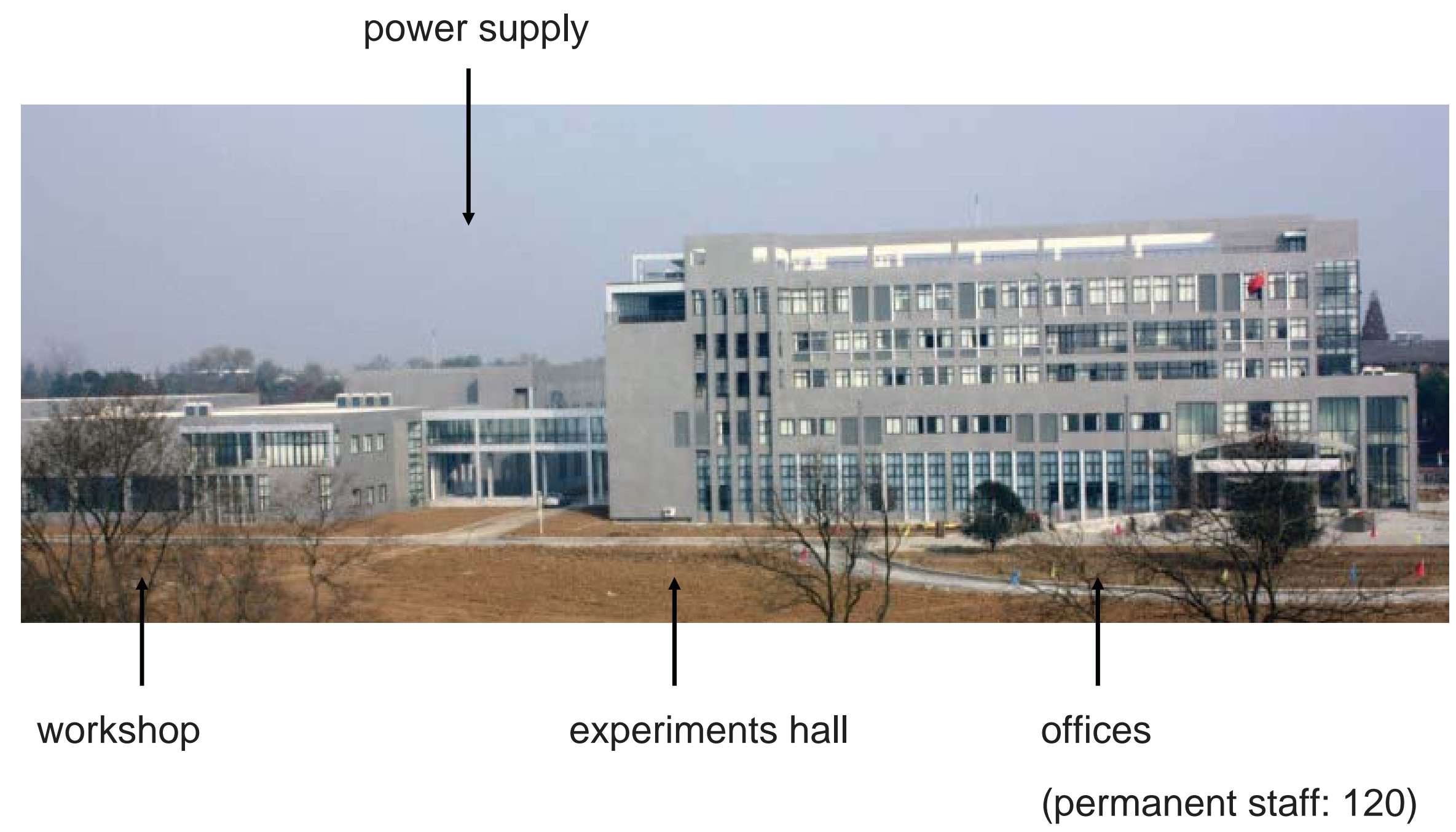




\section{International user community: LNCMI en 2011}

Number of proposals in 2011 , by nationality of PI

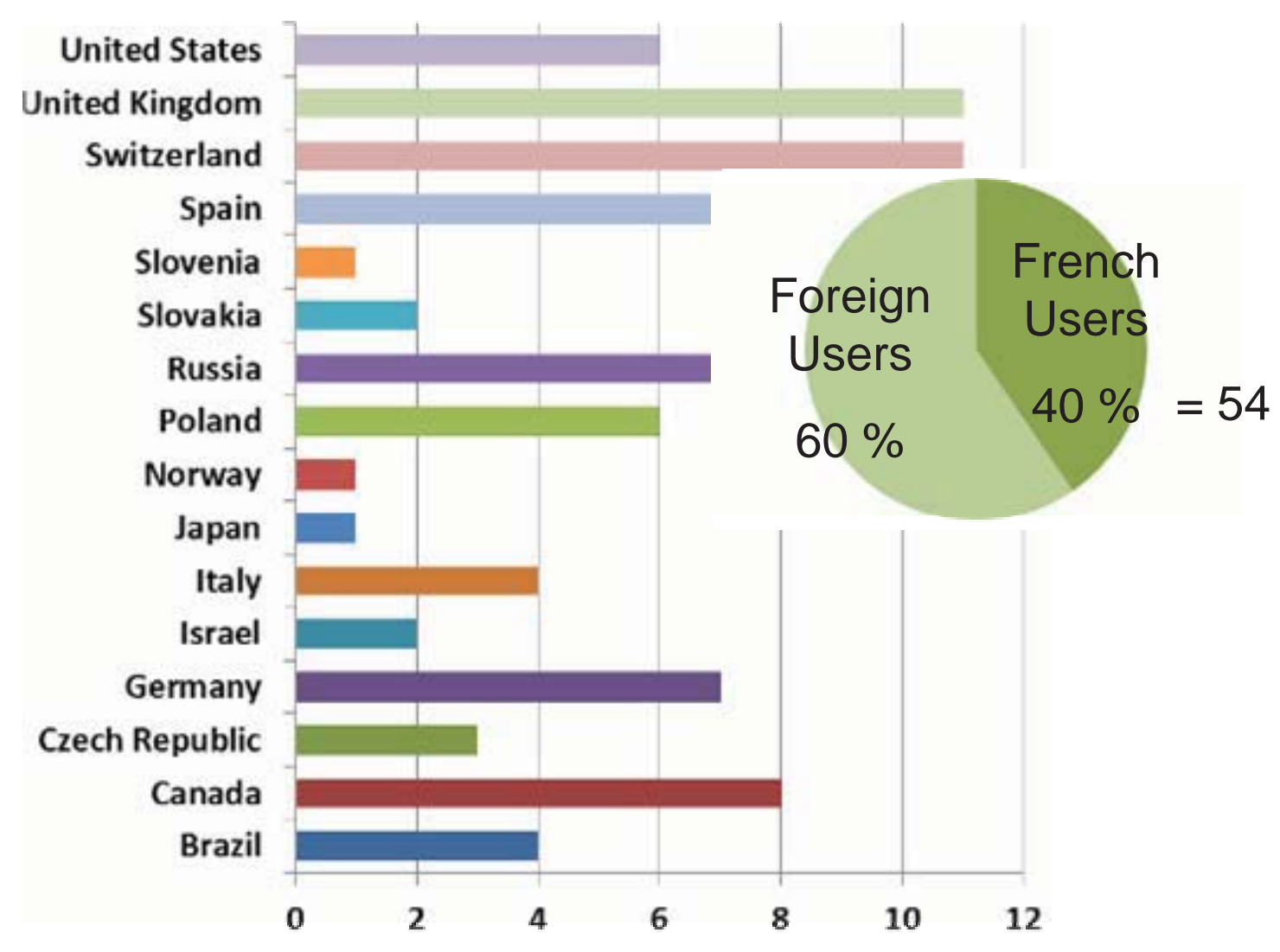


Traditionally, high magnetic fields are used in condensed matter science

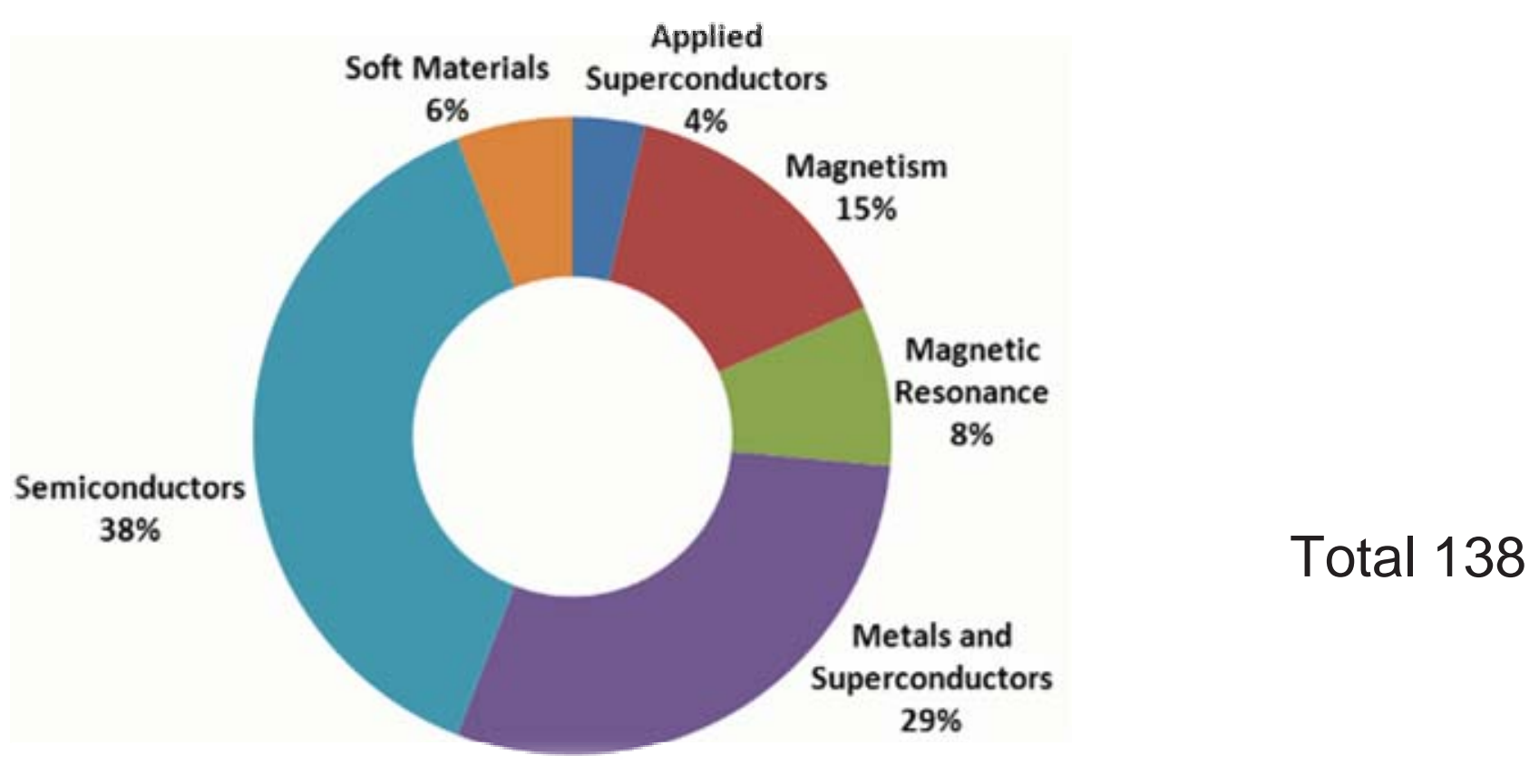

LNCMI 2011 


\section{ARTICLES}

Angle dependence of quantum oscillations in $\mathrm{YBa}_{2} \mathrm{Cu}_{3} \mathrm{O}_{6.59}$ shows free-spin behaviour of quasiparticles

B. J. Ramshaw ${ }^{1}$, Baptiste Vignolle ${ }^{2}$, James Day ${ }^{1}$, Ruixing Liang ${ }^{1,3}$, W. N. Hardy ${ }^{1,3}$, Cyril Proust ${ }^{2,3}$ and D. A. Bonn ${ }^{1,3 \star}$
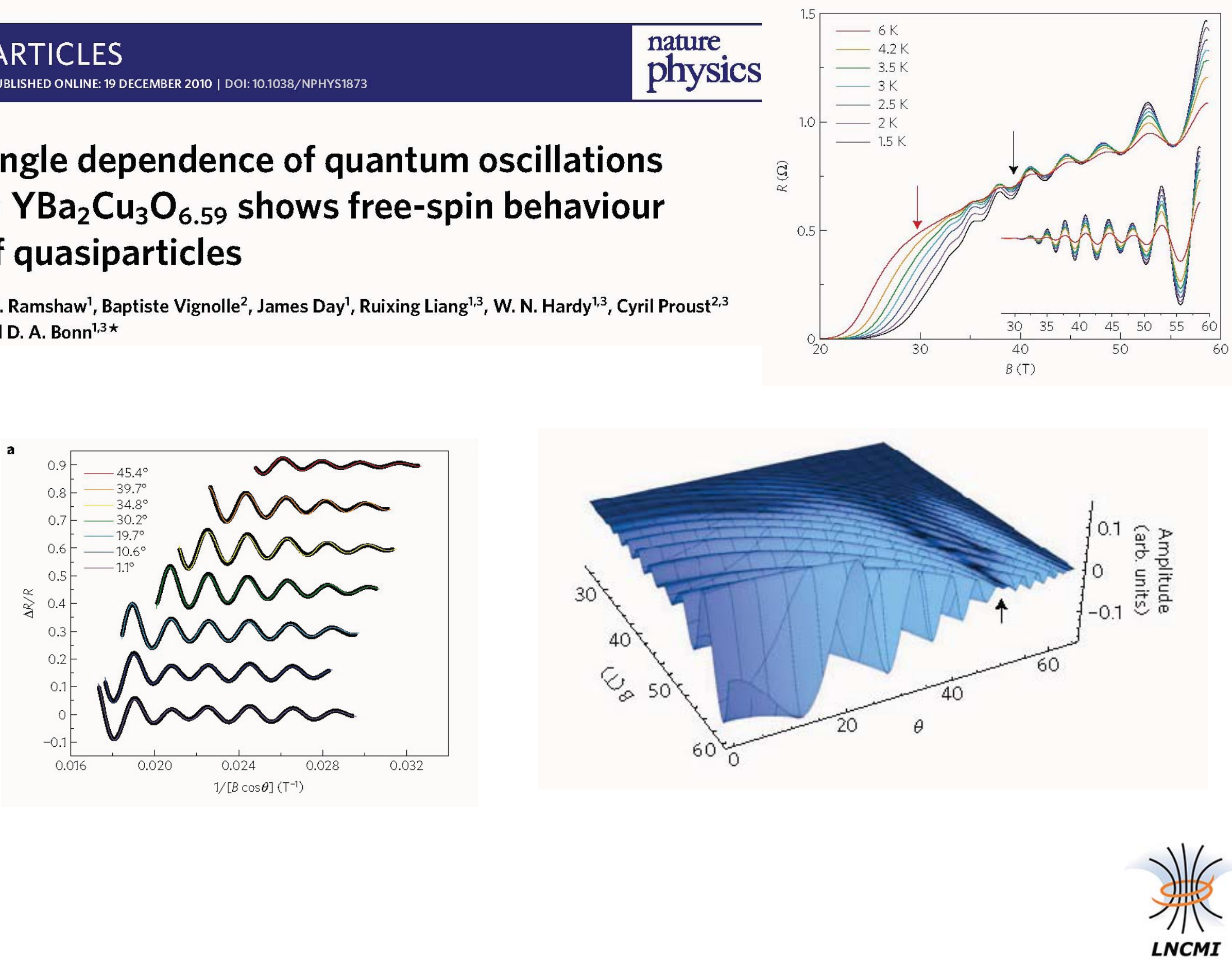
Stripe-induced Fermi pockets in $\mathrm{YBa}_{2} \mathrm{Cu}_{3} \mathrm{O}_{\mathbf{y}} \quad$ Wu et al, Nature 477, 191 (2011)

- Fermi surface reconstruction from Quantum Oscillation data (LNCMI-T)

- High field Cu NMR LNCMI-G:
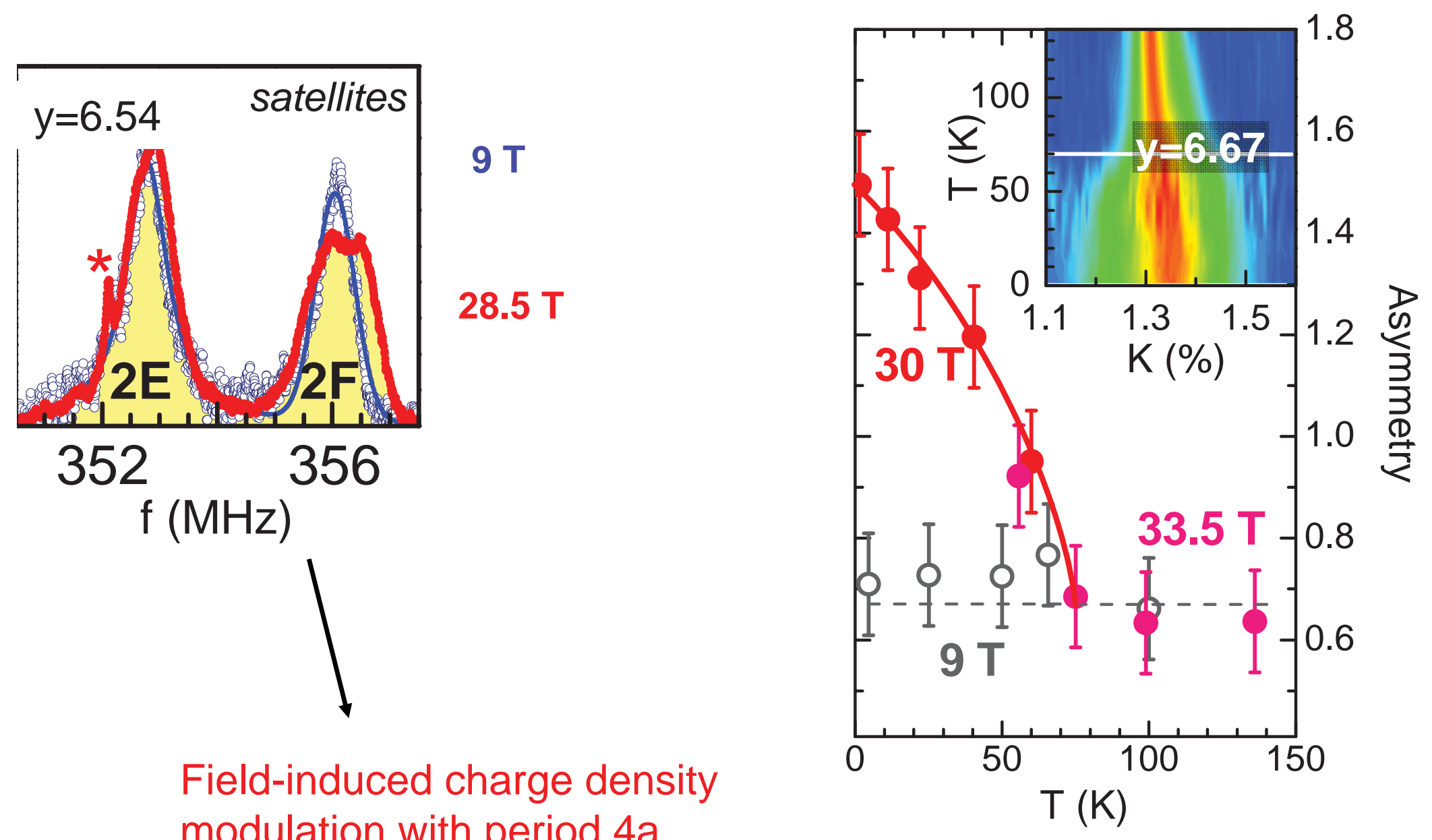

Field-induced charge density modulation with period $4 \mathrm{a}$, correlated with FS reconstruction 


\section{EuroMagNET2 FP7-I3: LNCMI +HLD + HFML}

A coordinated approach to access, experimental development and scientific exploitation of all European large infrastructures for high magnetic fields

\begin{tabular}{|c|c|c|c|c|c|c|c|}
\hline $\begin{array}{l}\text { WP } \\
\text { Number }\end{array}$ & Work package title & $\begin{array}{l}\text { Type of } \\
\text { activity }\end{array}$ & $\begin{array}{c}\text { Lead } \\
\text { participant } \\
\text { Number }\end{array}$ & $\begin{array}{c}\text { Lead } \\
\text { participant } \\
\text { short name }\end{array}$ & $\begin{array}{c}\text { Start } \\
\text { month }\end{array}$ & $\begin{array}{l}\text { End } \\
\text { month }\end{array}$ & $\begin{array}{c}\text { EC } \\
\text { contribution } \\
(\mathrm{M} €)\end{array}$ \\
\hline WP 1 & Consortium management & MGT & 1 & CNRS & 1 & 48 & 0,4 \\
\hline WP 2 & Networking Activities & CORD & 3 & FZD & 1 & 48 & 0,9 \\
\hline WP 3 & Transnational access static fields CNRS & SUPP & 1 & CNRS & 1 & 48 & 2,15 \\
\hline WP 4 & Transnational access pulsed fields CNRS & SUPP & 1 & CNRS & 1 & 48 & 0,65 \\
\hline WP 5 & Transnational access static fields $\mathrm{RU}$ & SUPP & 2 & $R U$ & 1 & 48 & 1,1 \\
\hline WP 6 & Transnational access pulsed fields FZD & SUPP & 3 & FZD & 1 & 48 & 0,3 \\
\hline WP 7 & $\begin{array}{l}\text { JRA High Field User Magnet Technology } \\
\text { and Operation }\end{array}$ & RTD & 1 & CNRS & 1 & 48 & 0,8 \\
\hline WP 8 & $\begin{array}{l}\text { JRA Nano-object measurements and local } \\
\text { spectroscopy }\end{array}$ & RTD & 2 & $R U$ & 1 & 48 & 0,6 \\
\hline WP 9 & JRA ES ${ }^{3}-N M R$ & RTD & 2 & $R U$ & 1 & 48 & 0,6 \\
\hline & TOTAL & & & & & & 7,5 \\
\hline
\end{tabular}




\section{ESRFI Project ‘ European Magnetic Field Laboratory’ (EMFL)}

Aim: offer to French and European scientist the same opportunities in high magnetic fields as in the USA

1) collaboration, specialisation and coordination between $L N C M I, H F M L$ and HLD.

2) Increase investments in Europe's high field facilities

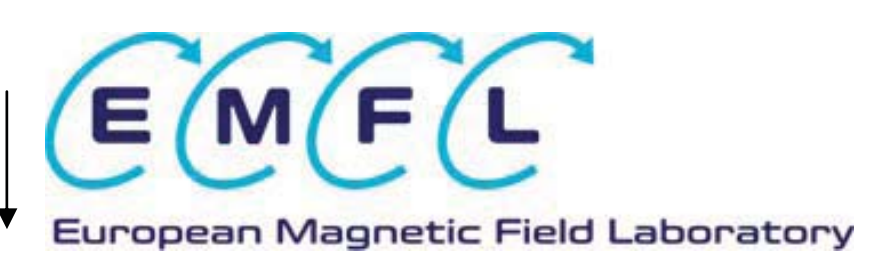

Intégration on the ESRFI Roadmap 2008

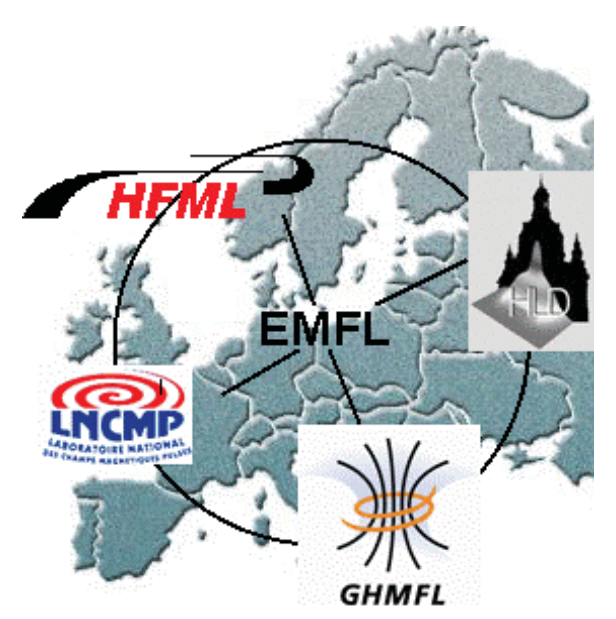




\section{ESFRI}

«The European Strategy Forum on Research Infrastructures was launched in April 2002. It brings together representatives of EU Member States and Associated States, appointed by Ministers in charge of Research, and one representative of the European Commission.

The role of ESFRI is to support a coherent approach to policy-making on research infrastructures in Europe, and to act as an incubator for international negotiations about concrete initiatives. «

- First ESRFI Roadmap in 2005, updated in 2008, updated 2010

- So far 240 projects submitted, 44 accepted on the Roadmap 


\section{The EMFL Preparatory Phase Proposal outline}

Identify legal and governance structure

Funding of investments and operation, staffing

Extension of the EMFL with other partners

Identifying and attracting new user communities

Roadmap for the technical and scientific evolution of the EMFL

Prototyping of new magnets/equipment

$\cdots \cdots$

Ready-to-sign founding contract

(Starting date 1/1/2011, duration 3 years, $4 \mathrm{M€}$ ) 
Magnetic fields are a small but well controlled and informative perturbation.

Generating high magnetic fields requires large investments and know how

The EMFL partners welcome requests/proposals for experiments in high magnetic fields.

Calls for proposals: every year May 15th, November 15th

www.emfl.eu

www.euromagnet2.eu
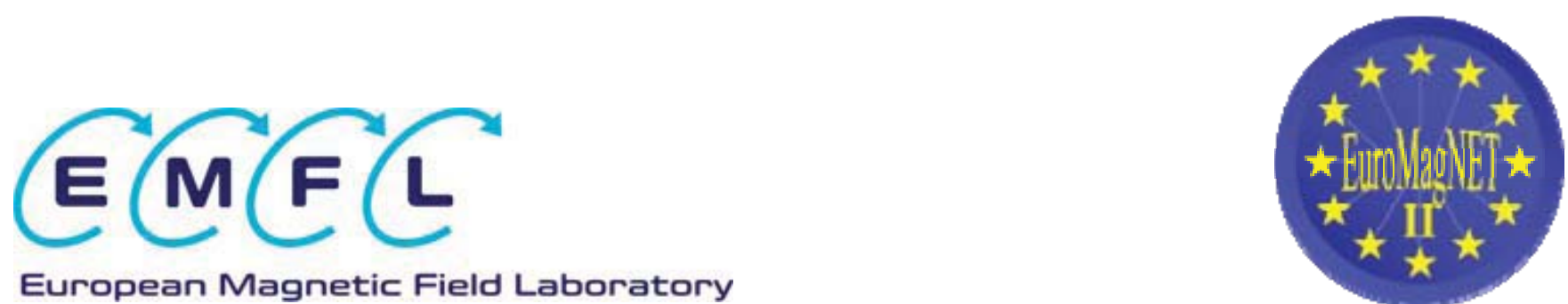